\title{
An Energetically Consistent Concurrent Multiscale Method For Heterogeneous Heat Transfer and Phase Transition Applications
}

\author{
Stephen Lin ${ }^{\mathrm{a}}$, Jacob Smith ${ }^{\mathrm{a}}$, Wing Kam Liu ${ }^{\mathrm{a}}$, Gregory J. Wagner ${ }^{\mathrm{a}, *}$ \\ ${ }^{a}$ Department of Mechanical Engineering, Northwestern University, Evanston, IL. USA
}

\begin{abstract}
A concurrent multiscale method is developed to model time-dependent heat transfer and phase transitions in heterogeneous media and is formulated in a way such that the energy being exchanged between scales is conserved. Ensuring this energetic consistency among scales enables the implementation of high fidelity physics-based models at critical locations within the coarse-scale to temporally and spatially resolve highly complex and localized phenomena. To achieve this, only Neumann boundary conditions are applied over the fine scale domain, ensuring a conservative formulation. The coarse-scale solution is used to reconstruct these Neumann boundary conditions on the fine scale, which are then used to evolve a separate system of governing equations. The results on the fine scale are then sent back to the coarse scale through an energy-based homogenization scheme. Transient simulations for the heat equation are implemented with the proposed method to demonstrate its accuracy in energy conservation and effectiveness, including the coupling of a phase field model at the fine scale to a coarse-scale heat equation.
\end{abstract}

Keywords: Concurrent multiscale method, Multilevel finite elements, Heat equation, Phase field models

1 1. Introduction

2

Preprint submitted to Elsevier 
To address this, high-fidelity fine scale models have been developed to provide the temporal and spatial resolution necessary to simulate phase transitions. However, the need to simulate physically relevant macroscale engineering systems introduces a sizable disparity between the length and time scales over which these processes occur and the computational domain. This disparity between the length and time scales enforces a considerable constraint on the size of the computational domain for a microscopic model so that the resulting computational costs of these models are no longer practical.

The aforementioned restrictions have motivated the development of multiscale modeling methods to offer a compromise between efficiency and accuracy. In these methods, multiple models at different time/length scales are coupled together to give a comprehensive description of a physical system. These models may have their physical basis derived from different laws, e.g. from molecular dynamics at the fine scale and from continuum mechanics at the coarse scale. A comprehensive review of the current state of the art multiscale methods and software can be found in the text written by Fish et al. [1]. Of these methods, much work has been devoted to the area of concurrent multiscale modeling because of its ability to exchange information between scales during the course of a simulation.

Multiple concurrent methods have been developed for coupling molecular dynamics (MD) and finite element (FE) simulations, otherwise known as atomistic/continuum coupling methods. For instance, Shenoy et al. developed a quasi-continuum method in which coupling between MD and FE is achieved by constraining a number of atoms in MD via the nodal displacement calculated by the FE solution [2]. Wagner and Liu proposed a method for coupling the atomistic and continuum scale by projecting a MD solution onto the coarse scale (FE) shape functions [3]. Xiao and Belytschko presented a method to reduce spurious wave reflections present at the molecular-continuum interface [4]. Chen and Fish [5, 6] and Fish et al. [7] applied the Generalized Mathematical Homogenization (GMH) theory to derive coarse-scale thermo-mechanical constitutive laws from the MD solution field. Lee and Basaran recently coupled FE with MD using a weighted average momentum principle [8]. These are just a sample of atomistic/continuum coupling methods that have been developed to incorporate localized information provided by MD simulations to continuum-based FE models. A comprehensive review for these class of methods is given by Miller et al. [9].

Considerable effort has also been focused on developing concurrent methods targeted at coupling multiple FE models at separate scales; one of these methods is the multigrid homogenization method. This method draws its inspiration from the multigrid iterative solver for elliptic equations [10, 11]. Early work in applying these iterative methods to the field of multiscale analysis have been done by Fish et al. $[12,13]$. In this method, an element within the coarse scale discretization is further refined to capture complex material heterogeneities on the fine scale. This particular method relies on the formulation of the projection and restriction operators that transfer information between the coarse and fine scale. Boffy et al. [14] developed a formulation for 
these operators to address high material property variations. Cecot et al. [15] utilized higher order Lagrange shape functions and bubble functions.

Another category of methods similar to the multigrid homogenization are the multiscale finite element methods (MsFEM). Rather than projecting the solution fields between scales, the coarse scale basis or shape functions are modified to assimilate information from the fine scale. This method has been further developed by modifying the linear basis functions [16] and introducing higher order basis functions $[17,18]$ to the coarse scale. Both the multigrid methods and MsFEM, however, implement the same physical model at the various scales, and are used mainly to account for geometrical details on the fine scale.

The Variational Multiscale (VMS) formulation proposed by Hughes et al. [19, 20], originally developed to address the origins of stabilized FE, has provided a generalized framework for fine scale modeling. This is achieved by a decomposition of the solution field into its coarse and fine scale components. The coarse scale is then projected into an appropriate subspace while the fine scale is directly stabilized against highly localized affects such as those seen in turbulence modeling. This method has been further developed in later work and applied to problems involving incompressible flows [21, 22, 23] and convection-diffusion [24, 25, 26].

A class of unit-cell based concurrent methods known as computational homogenization have been widely used for modeling mechanical response of materials with complex microstructure [27, 28, 29]. In this multilevel FE analysis, a fine scale representative volume element (RVE) representing a periodic microstructure is constructed around each Gauss integration point within the coarse scale domain. At each integration point, a coarse scale constant strain is evaluated and applied over a unit cell as Dirichlet periodic boundary conditions to solve mechanical equations for an effective stress response [30, 31]; finite element simulations are used at both scales, resulting in the socalled $\mathrm{FE}^{2}$ method. Higher order approximations for the coarse scale strain evaluations have been developed by Kouznetsova et al. [32] in order to resolve non-uniform macroscopic deformation fields and account for microstructural size. Non-periodic responses such as crack growth and initiation have been modeled by this class of methods by neglecting any periodic assumptions [33]. These computational homogenization methods have also been applied to heat transfer problems, where a coarse-scale transient formulation extracts a heat flux and conductivity from the solution of a fine-scale analysis $[34,35,36]$. These methods have typically used a quasi-static assumption at the fine scale, where inertia or heat capacity is neglected on the RVE computation. An exception is the recent work of Sridhar et al. [37], who considered inertial effects on the fine scale for locally resonant acoustic metamaterials. In that case a reduced order model was developed to accelerate the fine scale simulations, which are evaluated at every integration point in the macroscale domain.

In a similar vein to the computational homogenization approach, $\mathrm{E}$ et al. [38, 39] developed the Heterogeneous Multiscale Method (HMM), a general framework used to design numerical methods incorporating multiscale and multiphysics phenomena. In this framework, a fine scale model is 
linked to select critical regions in the coarse scale with different physical models being applied to each scale. The coarse and fine scales are coupled by utilizing the coarse scale solution to provide constraints for setting up the fine scale models. The coupling between individual scales is established through having the coarse scale solution provide constraints for setting up the fine scale model; the resulting solution field can then be used to reconstruct information missing on the coarse scale, such as the stiffness matrix, flux or force vectors.

The aforementioned unit-cell and multigrid based simulation methods typically use a fine scale simulation to compute a stress or flux given a specific set of boundary conditions obtained from the coarse scale. In effect the fine scale provides a way of computing a constitutive model. The applied boundary conditions most often take the form of periodic conditions on the fluctuation about a linear field (see for example [34]), which preserves the correct mean and gradient between the macro and micro scales. However, in our current approach we are interested in problems in which the rate of change of the energy of the fine scale domain is non-negligible (for example, in the case of a moving phase boundary at which latent heat is absorbed or released). Furthermore, we desire to track the evolution of the fine scale fields in detail, and not just to compute an effective constitutive model. For this case, periodic boundary conditions on the fine scale, even on the fluctuation about a linear temperature field, lead to zero rate of change in the average energy of the fine scale; this can be demonstrated by integrating the energy equation over the fine scale domain and assuming periodic boundary conditions (see Section 2.3). In order to capture a non-zero rate of change, a more effective approach is to apply Neumann boundary conditions with a non-constant applied flux. In this way the rate of change of energy can be made consistent between the macro and micro scales.

The necessity for a non-constant (in space) applied flux on the boundaries of the fine scale further restricts the way in which the macro and micro scales are coupled. In the $\mathrm{FE}^{2}$ and similar methods, the fine scale domain is coupled to the solution at an integration point in the coarse scale simulation. However, if linear finite elements are used for the macro scale, the flux is constant on the interior of the element, and is discontinuous at element boundaries and nodes. In order to solve a fine scale domain to simulate a time-varying energy, we must construct an applied boundary flux that corresponds, in some sense, to the rate of change of the coarse scale. In this paper we show that an effective and accurate approach is to use the discrete divergence of the flux at a coarse scale node to construct a fine scale boundary condition, leading to a consistent energy change.

In the remainder of this work we develop a concurrent multiscale method designed to ensure energy conservation between scales. This method is targeted at coupling transient phase transition models that may be different at each scale. We use the fine scale domain to represent a nodal point, rather than an integration or quadrature point, in the macroscale. This choice allows us to capture the effects of macroscale fluxes that vary across the point of interest, leading to time-dependent behavior. To avoid the computational expense of evaluating the fine scale simulation everywhere, we 
evaluate it only at one or a small number of select locations, and elsewhere use an analytical or offline computational homogenization to represent the microstructural effects. By applying Neumann boundary conditions derived from the coarse-scale solution at individual nodes, we show that we are able to achieve consistency between the coarse and fine scale energy evolution. In this work, we follow the methodology presented by the HMM; high fidelity multiphysics models are implemented within the fine scale in order to resolve extremely localized physical phenomena at select coarse scale nodes while continuum-based models are applied at all other regions. We combine this approach with that employed by $\mathrm{FE}^{2}$ methods and use the coarse scale solution field as well as its gradients to construct boundary conditions to evolve the fine scale models governing a unit cell constructed over select coarse scale nodes.

In this work, we follow the methodology presented by the HMM; high fidelity multiphysics models are implemented within the fine scale in order to resolve extremely localized physical phenomena at select coarse scale nodes while continuum-based models are applied at all other regions. We combine this approach with that employed by $\mathrm{FE}^{2}$ methods and use the coarse scale solution field as well as its gradients to construct boundary conditions to evolve the fine scale models governing a unit cell constructed over select coarse scale nodes. However, these fine scale boundary conditions are formulated such that the energy evolves over the fine scale to match the energy evolution of the coarse scale node it is coupled to.

In Section 2, we provide the formulation of our proposed concurrent method. Section 3 demonstrates our proposed method's capability to maintain energetic consistency between the two scales as well as an application for modeling solidification processes. Concluding remarks are given in Section 4 .

\section{Formulation of the Energetically Consistent Concurrent Method}

In this section, we present our method for linking the coarse and fine scales together using the heat equation as an example. We denote the coarse and fine scales as $\Omega$ and $\omega$, respectively. The domain $\omega$ is constructed by taking a control volume centered around a node of interest instead of an integration point within the coarse scale domain, as seen in Figure 1, and will be discretized with an independent mesh. Our choice for choosing the $\omega$ to be linked to a node rather than an integration point is motivated by the fact that we want to capture transient effects on the fine-scale. For linear finite elements, the heat flux would be constant across $\omega$ if it was linked to an integration point, and thus the average fine-scale energy would not change over time. Only gradients in the heat flux field (which is a flux difference across $\omega$ ) will internally drive a change in energy over time. We can achieve this flux difference across $\omega$ by linking it to a node, where the heat flux is discontinuous. 


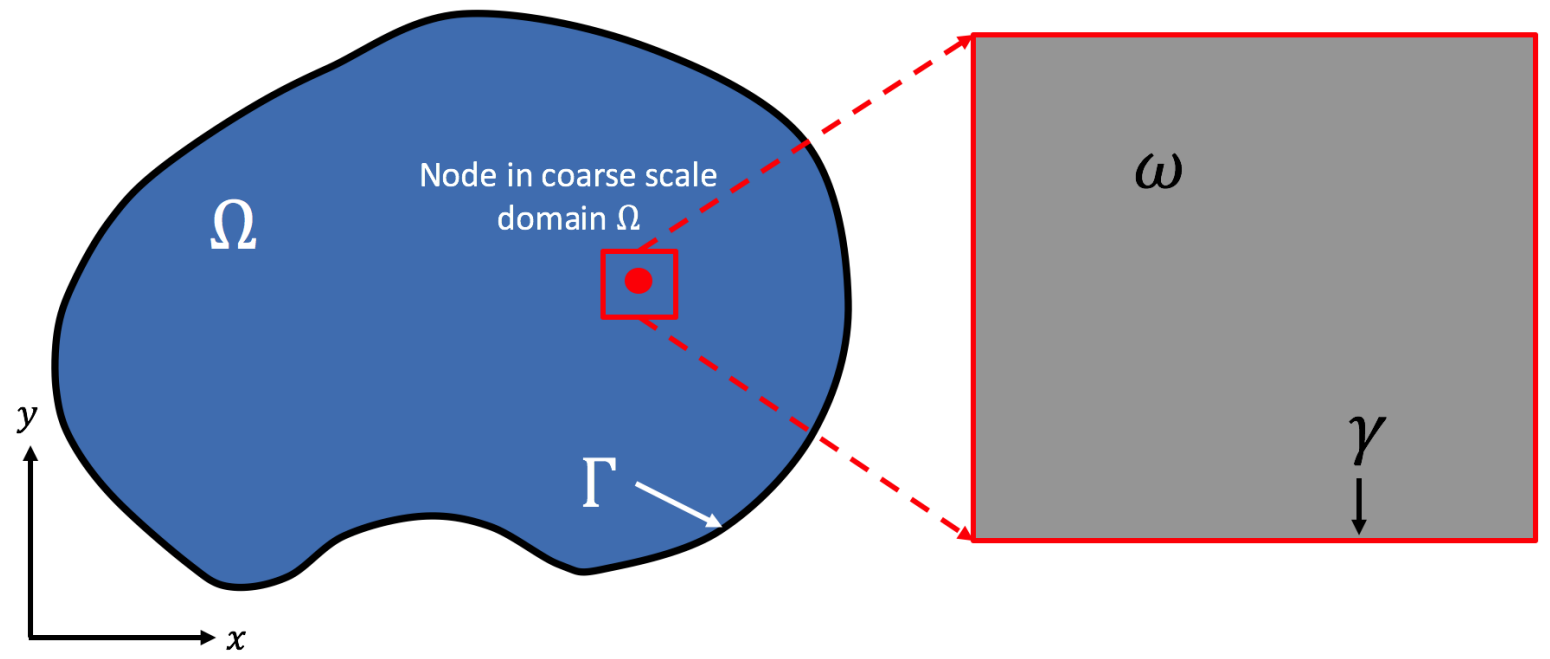

Figure 1: Coarse scale domain $\Omega$ and fine scale domain $\omega$ centered around a node in $\Omega$

\subsection{Finite Element Formulation of the Coarse-Scale Equations}

The strong form of the heat equation on the coarse scale $\Omega$ takes the following form

$$
\begin{array}{r}
-\nabla \cdot \overline{\boldsymbol{q}}+S=\bar{\rho} \bar{c}_{p} \dot{T} \text { in } \Omega \\
T=\hat{T} \text { on } \Gamma_{D} \\
-\overline{\boldsymbol{q}} \cdot \overline{\boldsymbol{n}}_{\Gamma}=\hat{q} \text { on } \Gamma_{N} \\
T(0, \boldsymbol{x})=T_{0}(\boldsymbol{x}) \text { in } \Omega
\end{array}
$$

Here, $T$ is the temperature with an over dot notation denoting its time derivative, $\boldsymbol{x}$ is a spatial coordinate, $\overline{\boldsymbol{q}}$ is the heat flux, and $S$ is a volumetric heat source term. The material properties $\bar{\rho}, \bar{c}_{p}$ and $\overline{\boldsymbol{K}}$ are the effective density, specific heat and thermal conductivity, respectively, and are obtained using a homogenization technique discussed in Section 2.3.4. (Note that where appropriate, we adopt a convention that properties and fields on the coarse scale are denoted with an overbar in order to distinguish them from their fine scale counterparts.) Additionally, the boundary $\Gamma$ is decomposed into regions where Neumann and Dirichlet boundary conditions are applied $(\Gamma=$ $\left.\Gamma_{D} \cup \Gamma_{N}\right)$. The parameters associated with the boundary are $\hat{T}, \hat{q}$ and $\overline{\boldsymbol{n}}_{\Gamma}$ which are the Dirichlet boundary temperature, Neumann boundary flux and unit outward normal of $\Gamma$, respectively. The initial condition is provided through a given initial temperature field $T_{0}(\boldsymbol{x})$.

Multiplying Eq. (1a) by a collection of weight functions $w$ and integrating over $\Omega$ results in:

$$
\int_{\Omega} w \bar{\rho} \bar{c}_{p} \dot{T} d \Omega=-\int_{\Omega} w \nabla \cdot \overline{\boldsymbol{q}} d \Omega+\int_{\Omega} w S d \Omega
$$

Utilizing Fourier's law of conduction $\overline{\boldsymbol{q}}=-\overline{\boldsymbol{K}} \nabla T$ and integration by parts on the first term on the 
right hand side of Eq. (2) results in

$$
\int_{\Omega} w \bar{\rho} \bar{c}_{p} \dot{T} d \Omega=-\int_{\Omega} \nabla w \cdot \overline{\boldsymbol{K}} \nabla T d \Omega+\int_{\Gamma_{N}} w \hat{q} d \Gamma+\int_{\Omega} w S d \Omega
$$

where $\overline{\boldsymbol{K}}$ is the coarse-scale thermal conductivity tensor. Additionally, we have used the Neumann boundary condition in Eq. (1c) for the integral boundary term $\int_{\Gamma_{N}} w \hat{q} d \Gamma$ and require the weight functions $w$ to vanish on the Dirichlet boundary $\Gamma_{D}$. The continuous solution $T(\boldsymbol{x}, t)$ is approximated by a finite element trial solution

$$
\begin{aligned}
T(\boldsymbol{x}, t) & =\sum_{I=1}^{\bar{n}_{N}} \bar{N}_{I}(\boldsymbol{x}) T_{I}(t) \\
\frac{\partial T(\boldsymbol{x}, t)}{\partial x_{i}} & =\sum_{I=1}^{\bar{n}_{N}} \frac{\partial \bar{N}_{I}(\boldsymbol{x})}{\partial x_{i}} T_{I}(t)=\sum_{I=1}^{\bar{n}_{N}} \bar{N}_{I, i}(\boldsymbol{x}) T_{I}(t)
\end{aligned}
$$

here, $\bar{N}_{I}(x)$ are the coarse-scale finite element shape functions and are $C^{0}$ interpolants, $T_{I}(t)$ are the coarse-scale nodal temperatures which are a function of time, and $\bar{n}_{N}$ is the number of coarse-scale nodes. Capital subscripts $(I)$ refer to node number and lowercase subscripts $(i)$ denote a spatial index. We use the comma notation to denote differentiation with respect to spatial indices. The weight functions $w$ are approximated in a similar manner:

$$
\begin{gathered}
w(\boldsymbol{x})=\sum_{I=1}^{\bar{n}_{N}} \bar{N}_{I}(\boldsymbol{x}) w_{I} \\
\frac{\partial w(\boldsymbol{x}, t)}{\partial x_{i}}=\sum_{I=1}^{\bar{n}_{N}} \bar{N}_{I, i}(\boldsymbol{x}) w_{I}(t)
\end{gathered}
$$

where $w_{I}$ are nodal values of the test function. Substituting the interpolations given by Eqs. (4) and (5) into (3) and invoking the arbitrariness of $w_{I}$ results in

$$
\sum_{J}^{\bar{n}_{N}} \int_{\Omega} \bar{\rho} \bar{c}_{p} \bar{N}_{I} \bar{N}_{J} d \Omega \dot{T}_{J}=-\sum_{J}^{\bar{n}_{N}} \int_{\Omega} \bar{N}_{I, i} K_{i j} \bar{N}_{J, j} T_{J} d \Omega+\int_{\Gamma_{N}} \bar{N}_{I} \hat{q} d \Gamma+\int_{\Omega} \bar{N}_{I} S d \Omega
$$

Here, repeated spatial indices imply a summation over the number of spatial dimensions. We perform a row-sum or mass-lumping technique on the left hand side mass matrix term in Eq. (6). Utilizing the partition of unity property for the finite element shape function results in

$$
\sum_{J}^{\bar{n}_{N}} \int_{\Omega} \bar{\rho} \bar{c}_{p} \bar{N}_{I} \bar{N}_{J} d \Omega \dot{T}_{J} \approx \int_{\Omega} \bar{\rho} \bar{c}_{p} \bar{N}_{I} d \Omega \dot{T}_{I}
$$


We let $C_{I}$ and $\Delta V_{I}$ denote the coarse-scale effective specific capacitance and volume at a node $I$, respectively, and make the following substitutions:

$$
\begin{aligned}
F_{I} & =-\sum_{J}^{\bar{n}_{N}} \int_{\Omega} \bar{N}_{I, i} K_{i j} \bar{N}_{J, j} T_{J} d \Omega+\int_{\Gamma_{N}} \bar{N}_{I} \hat{q} d \Gamma \\
S_{I} & =\int_{\Omega} \bar{N}_{I} S d \Omega \\
\Delta V_{I} C_{I} & =\int_{\Omega} \bar{\rho} \bar{c}_{p} \bar{N}_{I} d \Omega
\end{aligned}
$$

where $S_{I}$ and $F_{I}$ are nodal contributions resulting from the volumetric source term and the heat flux divergence, respectively. Substituting the expressions given by Eqs. (7) and (8) results in a set of equations calculating the energy rate at a node $I$ :

$$
C_{I} \dot{T}_{I}=\frac{1}{\Delta V_{I}}\left(F_{I}+S_{I}\right)
$$

These nodal equations will provide information for constructing the fine scale system of equations such that the energy rate throughout $\omega$ will match the energy rate for a node $I$.

\subsection{Finite Element Formulation for the Fine-scale Equations}

The strong form for the fine scale domain takes on a form similar to the coarse scale with the exception of the enforced boundary conditions:

$$
\begin{array}{r}
-\nabla \cdot \boldsymbol{q}+s=\rho c_{p} \dot{\theta} \text { in } \omega \\
-\boldsymbol{q} \cdot \boldsymbol{n}=q_{n} \text { on } \gamma \\
\theta(0, \boldsymbol{x})=\theta_{0}(\boldsymbol{x}) \text { in } \omega
\end{array}
$$

Here, $\theta$ is the temperature, $\boldsymbol{q}$ is the heat flux, $s$ is a volumetric heat source term, $\rho$ is the material density, $c_{p}$ is the material specific heat, $\boldsymbol{n}$ is a unit outward normal of $\gamma$ and $q_{n}$ is the prescribed flux for a Neumann boundary condition on $\gamma$. The initial condition is given by $\theta_{0}(\boldsymbol{x})$. Unlike the coarse-scale formulation, the fine scale will not enforce a Dirichlet boundary condition on $\gamma$. Our choice for this will become clear in the following sections. We now take a finite element formulation of the fine-scale strong form equation expressed in Eq. (10a). Multiplying Eq. (10a) by a collection of weight functions $v$ and integrating over $\omega$ results in

$$
\int_{\omega} v \rho c_{p} \dot{\theta} d \omega=-\int_{\omega} v \nabla \cdot \boldsymbol{q} d \omega+\int_{\omega} v s d \omega
$$


Using Fourier's law of conduction $\boldsymbol{q}=-\kappa \nabla \theta$ and the divergence theorem on the second term on the right hand side of Eq. (11) results in

$$
\int_{\omega} v \rho c_{p} \dot{\theta} d \omega=-\int_{\omega} \nabla v \cdot \kappa \nabla \theta d \omega+\int_{\gamma} v \boldsymbol{q} \cdot \boldsymbol{n} d \gamma+\int_{\omega} v s d \omega
$$

were we have assumed that the material properties within $\omega$ are isotropic, thus a scalar thermal conductivity $\kappa$ is used. The continuous solution $\theta(\boldsymbol{x}, t)$ is approximated by a finite element trial solution

$$
\begin{aligned}
\theta(\boldsymbol{x}, t) & =\sum_{I=1}^{n_{N}} N_{I}(\boldsymbol{x}) \theta_{I}(t) \\
\frac{\partial \theta(\boldsymbol{x}, t)}{\partial x_{i}} & =\sum_{I=1}^{n_{N}} N_{I, i}(\boldsymbol{x}) \theta_{I}(t)
\end{aligned}
$$

Here, $N_{I}$ are the fine-scale finite element shape functions and are $C^{0}$ interpolants, $\theta_{I}(t)$ are the fine scale nodal temperatures which are a function of time and $n_{N}$ is the number of fine-scale nodes. The weight functions $v$ are approximated in a similar manner

$$
\begin{aligned}
v(\boldsymbol{x}, t) & =\sum_{I=1}^{n_{N}} N_{I}(\boldsymbol{x}) v_{I}(t) \\
\frac{\partial v(\boldsymbol{x}, t)}{\partial x_{i}} & =\sum_{I=1}^{n_{N}} N_{I, i}(\boldsymbol{x}) v_{I}(t)
\end{aligned}
$$

Inserting the interpolations defined in Eqs. (13) and (14) into the fine-scale weak form equation (12) and invoking the arbitrariness of $v_{I}$ results in

$$
\sum_{J}^{n_{N}} \int_{\omega} \rho c_{p} N_{I} N_{J} d \omega \dot{\theta}_{J}=-\sum_{J}^{n_{N}} \int_{\omega} \kappa N_{I, i} N_{J, i} \theta_{J} d \omega+\int_{\gamma} N_{I} q_{i} n_{i} d \gamma+\int_{\gamma} N_{I} s d \omega
$$

\subsection{Coupling the Coarse-scale and Fine-scale Equations}

To establish a relationship between the fine scale and coarse scale, we return to the fine-scale weak formulation Eq. (12). This requires the specification of boundary conditions on the fine scale domain in terms of the coarse scale quantities. One approach often taken in multi-level finite element schemes (see for example [34]) is to write the fine scale field, in this case the temperature, as the sum of a linear field plus a field that fluctuates on the fine scale: $\theta=\theta_{c}+(\nabla \theta)_{c} \cdot\left(\mathbf{x}-\mathbf{x}_{0}\right)+\theta_{f}(\mathbf{x})$. Here, $\theta_{c}$ and $(\nabla \theta)_{c}$ are coarse scale quantities assumed to be constant on the fine scale, and $\mathbf{x}_{0}$ is 
the center or origin of the fine scale domain. Often, periodic boundary conditions are applied to the fluctuating field $\theta_{f}(\mathbf{x})$. However, this choice leads to zero rate of change in the fine scale energy in the absence of source terms when the thermal conductivity is also periodic. To see this, we can evaluate Eq. (12) with $v=1, s=0$, and $\boldsymbol{q}=-k \nabla \theta$ to get:

$$
\int_{\omega} \rho c_{p} \dot{\theta} d \omega=-(\nabla \theta)_{c} \cdot \int_{\gamma} k \mathbf{n} d \gamma-\int_{\gamma} k \nabla \theta_{f} \cdot \mathbf{n} d \gamma=0
$$

where the two integrals over $\gamma$ are zero because of the periodicity of $k$ and $k \nabla \theta_{f}$, respectively.

Because we require a non-zero change in the average fine scale energy, in this work we instead enforce Neumann boundary conditions on the fine scale domain. With this choice, the test functions $v$ do not vanish anywhere on $\gamma$, and Eq. (12) is in conservative form. To illustrate this, we set $v=1$ in (12):

$$
\int_{\omega} \rho c_{p} \dot{\theta} d \omega=\int_{\gamma} \boldsymbol{q} \cdot \boldsymbol{n} d \gamma+\int_{\omega} s d \omega
$$

Eq. (17) is in an integral conservative form, which simply states that a rate change in quantity (energy in this case) over an arbitrary volume $\omega$ is equal to the fine-scale heat flux $\boldsymbol{q}$ entering and leaving through its boundary $\gamma$ plus any volumetric sinks or sources acting over $\omega$. Enforcing Dirichlet boundary conditions on $\gamma$ would not satisfy a conservative integral form since portions of $\gamma$ would be excluded due to constraints imposed by $v$.

In addition, it can be seen that the energy evolution of the coarse-scale system is governed by the energy evolution at the nodes given by Eq. (9). We need to ensure that the energy evolution over the fine-scale domain given by Eq. (17) matches that at the node given by (9). Doing so will ensure that we will conserve energy over the coarse-scale system. The coarse-scale nodal equation given in Eq. (9) has units of energy rate per unit volume. In order to match this nodal energy rate to the fine scale domain, Eq. (9) is scaled by the volume of the fine scale domain $|\omega|$. The scaled coarse-scale nodal energy evolution and the conservative fine-scale energy evolution equations are reiterated below

$$
\begin{aligned}
|\omega| C_{I} \dot{T}_{I} & =\frac{|\omega|}{\Delta V_{I}}\left(F_{I}+S_{I}\right) \\
\int_{\omega} \rho c_{p} \dot{\theta} d \omega & =\int_{\gamma} \boldsymbol{q} \cdot \boldsymbol{n} d \gamma+\int_{\omega} s d \omega
\end{aligned}
$$

We create a multiscale coupling scheme by matching each individual coarse-scale term in Eq. (18a) with its respective fine-scale counterpart in Eq. (18b). In our formulation, we will implement an 
explicit time integrator for the rate terms $\dot{\theta}$ and $\dot{T}$ and denote any variables superscripted with $n$ and $n+1$ to be the variable at a temporal state $t^{n}$ and $t^{n+1}$, respectively.

\subsubsection{Energetic Homogenization of the Fine-scale Solution}

We first equate the left hand side terms of Eqs. (18a) and (18b), which results in

$$
|\omega| C_{I} \dot{T}_{I}=\int_{\omega} \rho c_{p} \dot{\theta} d \omega
$$

Dividing $C_{I}$ and $|\omega|$ through on both sides and substituting the definition of $C_{I}$ from Eq. (8c) yields

$$
\dot{T}_{I}=\frac{\int_{\omega} \rho c_{p} \dot{\theta} d \omega}{|\omega| C_{I}}=\frac{\int_{\omega} \rho c_{p} \dot{\theta} d \omega \Delta V_{I}}{|\omega| \int_{\Omega} \bar{\rho} \bar{c}_{p} \bar{N}_{I} d \Omega}
$$

Eq. (20) provides a formula for homogenizing or upscaling the temperature rate of the fine-scale domain $\omega$ into a node $I$. We can extend this formulation for homogenizing the temperature distribution of the fine-scale domain by taking the non-rate form of Eq. (20), resulting in

$$
T_{I}=\frac{\int_{\omega} \rho c_{p} \theta d \omega \Delta V_{I}}{|\omega| \int_{\Omega} \bar{\rho} \bar{c}_{p} \bar{N}_{I} d \Omega}
$$

This expression provides our method for homogenizing the fine-scale temperature distribution to a coupled node $I$.

\subsubsection{Reconstructing the Fine-scale Flux from the Coarse-scale Solution}

We now equate the first terms on the right hand side of Eqs. (18a) and (18b). The case considered in this section is for a coarse-scale node $I$ that is on the interior of $\Omega$. Using the definition of $F_{I}$ from Eq. (8a) and neglecting the boundary integral term results in

$$
\int_{\gamma} \boldsymbol{q} \cdot \boldsymbol{n} d \gamma=\frac{|\omega|}{\Delta V_{I}}\left(\sum_{J}^{\bar{n}_{N}} \int_{\Omega} \bar{N}_{I, i} K_{i j} \bar{N}_{J, j} T_{J} d \Omega\right)
$$

The right hand side of Eq. (22) represents the divergence of the coarse-scale nodal flux $\overline{\boldsymbol{q}}$. Note that in general the flux $\overline{\boldsymbol{q}}=-\overline{\boldsymbol{K}} \nabla T$ is discontinuous at element boundaries, so that the divergence of the flux is undefined at element boundaries, including node locations. The right hand side of Eq. (22), however, gives the weak-form contribution of the flux divergence to the energy change at the node. This quantity is well-defined and can be used to enforce consistency between the coarse and fine scales by reconstructing a fine-scale flux $\boldsymbol{q}$ that satisfies Eq. (22).

For convenience we define the nodal flux divergence $d_{I}$ as 


$$
d_{I}=\frac{1}{\Delta V_{I}}\left(\sum_{J}^{\bar{n}_{N}} \int_{\Omega} \bar{N}_{I, i} K_{i j} \bar{N}_{J, j} T_{J} d \Omega\right)
$$

We enforce two assumptions that allow us to uniquely reconstruct the fine-scale flux field $\boldsymbol{q}$ :

1. The fine-scale flux $\boldsymbol{q}$ is parallel to the coarse-scale nodal flux $\overline{\boldsymbol{q}}_{I}$ and only varies in the direction of $\overline{\boldsymbol{q}}_{I}$

2. The fine-scale flux $\boldsymbol{q}$ varies linearly over the fine-scale boundaries

It should be noted here that assumption 2 is used to enforce that the surface-averaged flux over the fine-scale boundaries reproduces the nodal flux at the coarse scale when using linear shape functions. Doing this ensures that the energy fluxes through fine scale boundaries will match the nodal energy rate through the coarse-scale node. The form of $\boldsymbol{q}$ that satisfies both these constraints along with $(22)$ is

$$
\boldsymbol{q}=\overline{\boldsymbol{q}}_{I}+d_{I}\left(\boldsymbol{x}-\boldsymbol{x}_{0}\right) \cdot \overline{\boldsymbol{n}}_{I} \overline{\boldsymbol{n}}_{I}
$$

where $\boldsymbol{x}$ is the coordinate of a point in $\omega, \boldsymbol{x}_{0}$ is the coordinate of the center of $\omega$, and $\overline{\boldsymbol{n}}_{I}$ is the unit vector of the coarse-scale nodal flux $\overline{\boldsymbol{q}}_{I}$. It is straightforward to show that the two assumptions above are satisfied by Eq. (24). Note that $\boldsymbol{q}$ need only be evaluated on the boundary $\gamma$, where it provides the Neumann boundary condition for the fine scale simulation.

\subsubsection{Reconstructing Source Terms on the Fine-scale Domain}

Finally, we equate the last term on the right hand sides of Eqs. (18a) and (18b) to construct the fine-scale source term.

$$
\int_{\omega} s d \omega=\frac{|\omega|}{\Delta V_{I}} S_{I}=\frac{|\omega|}{\Delta V_{I}} \int_{\Omega} N_{I} S d \Omega
$$

here we have utilized the definition of $S_{I}$ in Eq. (8b). Eq. (25) essentially scales the coarse-scale nodal force due to the source $S$ by a volumetric ratio and is distributed throughout $\omega$ as a constant source or sink term.

\subsubsection{Homogenization of Surrounding Coarse Scale Properties}

The fine scale domain may spatially resolve complex heterogeneous microstructures that have an assumed periodicity with varying material properties; numerically resolving these heterogeneities at each integration point on the coarse scale will result in an impractical computational expense. As a result, the surrounding coarse-scale domain that is not linked to the fine-scale requires additional 
treatment of the material properties. For the specific examples we have chosen, we utilize a semianalytical homogenization approach [40] to compute effective properties to resolve any material heterogeneities.

The coarse-scale capacitance, $\bar{\rho} \bar{c}_{p}$, can be calculated as a volume average over $\omega$ :

$$
\bar{\rho} \bar{c}_{p}=\frac{1}{|\omega|} \int_{\omega} \rho(x) c_{p}(x) d \omega
$$

The coarse-scale conductivity matrix $\boldsymbol{K}$ requires a more involved treatment for homogenization. We follow the method of homogenization for heat transfer applications outlined by Kamiński [40], which assumes periodic material heterogeneities over $\omega$, to calculate the coarse-scale conductivity matrix. A 2 step process is required to compute the coefficients of $\overline{\boldsymbol{K}}$, where each step solves a steady state problem. The first step solves for a smooth periodic solution $a_{j}$ in each spatial dimension

$$
\nabla \cdot\left(\kappa \nabla a_{j}\right)=-\frac{\partial \kappa}{\partial x_{j}}
$$

Here, we assume that $\kappa(x)$ now varies in space, and $\nabla_{j}=\frac{\partial}{\partial x_{j}}$ is the $j^{\text {th }}$ component of the gradient operator. Equation 27 is solved using the finite element method. The finite element solution field $a_{j}^{h}$ contains a periodic boundary condition on all boundaries. A standard weak formulation is taken by multiplying equation 27 with a test function $v$ and integrating over $\omega$.

$$
\int_{\omega} v \nabla \cdot\left(\kappa(x) \nabla a_{j}^{h}\right) d \omega=-\int_{\omega} v \frac{\partial \kappa}{\partial x_{j}} d \omega
$$

In applications involving heterogeneous materials, the conductivity $\kappa$ is discontinuous, thus its gradient is undefined. To address this, we apply integration by parts on both the left and right hand side terms in equation (28), resulting in

$$
\int_{\omega} \nabla v \cdot\left(\kappa(x) \nabla a_{j}^{h}\right) d \omega=-\int_{\omega} \frac{\partial v}{\partial x_{j}} \cdot \kappa d \omega
$$

The second step involves using the periodic solution $a_{j}^{h}$ to calculate the individual components of the coarse-scale conductivity tensor $K_{i j}$.

$$
K_{i j}=\left(\frac{1}{|\omega|} \int_{\omega} \kappa(x) d \omega\right) \delta_{i j}+\frac{1}{|\omega|} \int_{\omega} \kappa(x) \frac{\partial a_{j}^{h}}{\partial x_{i}} d \omega
$$

These coarse-scale material properties $\overline{\boldsymbol{K}}$ and $\bar{\rho} \bar{c}_{p}$ are substituted back into the coarse-scale finite element equation (3). It should be noted that for phase transition applications, these material heterogeneities may vary with time. Therefore, if material properties are different within each 
phase, then it becomes necessary to recompute the effective properties $\overline{\boldsymbol{K}}$ and $\bar{\rho} \bar{c}_{p}$ after each finescale update.

\subsection{Complete Concurrent Multiscale Methodology}

With the formulations provided in sections 2.3.1, 2.3.2 and 2.3.3, we can formulate a sub-cycling algorithm for our proposed concurrent multiscale method, which is outlined in the following steps:

1. Create a fine-scale domain $\omega$ over a coarse-scale node $I$ of interest

2. Construct the fine-scale flux field $\boldsymbol{q}$ using the coarse-scale nodal solution at time $t^{n}$ and Eq. (24) to evaluate the boundary integral term in Eq. (12) applied over $\gamma$

3. Use the coarse-scale nodal source term at time $t^{n}$ to calculate fine-scale source integral with Eq. (25)

4. Run a fine scale transient simulation over $\omega$ to evolve Eq. (12) from time $t^{n}$ to $t^{n+1}$

5. Upscale the results from the fine scale to the coarse scale using Eq. (21)

6. Perform a calculation over $\Omega$ to update all other coarse scale nodes from $t^{n}$ to $t^{n+1}$

7. Go back to step 2 until the entire simulation is finished

A sub-cycling routine takes place at step 4 to evolve the fine scale simulation. For the following examples, we utilize an explicit time integrator on both the fine and coarse scale. The coarse and fine scale time step will be denoted as $\Delta \bar{t}$ and $\Delta t$, respectively. The following section presents examples to demonstrate the capabilities of the proposed methodology.

\section{Numerical Examples}

A series of numerical experiments are performed to demonstrate the effectiveness of our proposed method:

1. 2D heat transfer comparing concurrent coupling between fine scale temperature and flux boundary conditions; homogeneous microstructure

2. 2D heat transfer with Neumann boundary conditions and a time-dependent source term; homogeneous microstructure

3. 2D heat transfer with Neumann boundary conditions and a time-dependent source term; periodic composite microstructure

4. 2D non-isothermal solidification; periodic composite microstructure

5. 3D heat transfer with Neumann boundary conditions; periodic composite microstructure 
Each test listed above is run twice, once with the fine-scale model sub-cycled with the coarsescale model and once with only the coarse-scale model using homogenized effective properties. The resultant temperature at the node of interest from the simulation that sub-cycles the fine-scale model will be denoted as $T_{\text {fine }}$ which is homogenized from the fine-scale solution field using Eq. (21); the resultant nodal temperature from the simulation that utilizes only the coarse-scale model with homogenized effective properties will be denoted as $T_{\text {coarse }}$. Additionally, $T_{\text {fine }}$ and $T_{\text {coarse }}$ will be tracked throughout the duration of each simulation to provide a measure of the energy rate throughout $\omega$ and the node of interest, respectively. A measure of the absolute error between these two values will be denoted as $E_{J}$, defined as

$$
E_{J}=\left|T_{\text {fine }}-T_{\text {coarse }}\right|
$$

$E_{J}$ will be used as a metric for energy conservation between the fine scale domain $\omega$ and the node of interest. Problems 1-4 are conducted using MATLAB [41], and Problem 5 is conducted using an inhouse thermal analysis finite element code written in Fortran 90. Problem 1 investigates the energy conservation capabilities of enforcing Dirichlet boundary conditions over the fine scale, which is utilized in most $\mathrm{FE}^{2}$ methods, and will be compared to the results when using our proposed method. Problem 2 demonstrates the consistent energy conservation between the fine scale domain $\omega$ and the node of interest with a rapidly changing nodal flux. Problem 3 shows our proposed method's ability to handle a spatially varying microstructure. Problem 4 exhibits our method's ability to couple a high resolution phase field model on the fine scale to a coarse scale approximation of phase transformation. Lastly, Problem 5 shows the application of our proposed method in a 3-dimensional setting. The material properties used for each problem are listed in Table 1

Table 1: Material properties listed for each Problem

\begin{tabular}{|c|c|c|c|c|c|}
\hline Parameter & Problem 1 & Problem 2 & Problem 3 & Problem 4 & Problem 5 \\
\hline$\rho_{0}$ & 1 & 1 & 0.1 & 1 & 1 \\
$\rho_{1}$ & - & - & 1.0 & - & - \\
$c_{p 0}$ & 1 & 1 & 0.1 & 1 & 1 \\
$c_{p 1}$ & - & - & 1 & - & - \\
$\kappa_{0}$ & 1 & 1 & 1 & 5 & 10 \\
$\kappa_{1}$ & - & - & 10 & 0.5 & 1 \\
\hline
\end{tabular}

\subsection{Comparison of Concurrent Coupling with Dirichlet and Neumann Boundary Conditions}

In this section, we justify our choice for constructing flux boundary conditions as opposed to temperature boundary conditions on the fine scale $\omega$. To compare our proposed method with a temperature $\mathrm{BC}$ approach, we will first develop a simple formulation for constructing temperature boundary conditions on $\omega$ from information given on $\Omega$. 


\subsubsection{Formulation for Temperature Boundary Conditions}

Figure 2a shows a sample fine scale domain $\omega$ constructed around a node $J$ within a patch of elements in the coarse scale domain $\Omega$. A total of 8 evaluation points within the patch of elements, marked with "X", are used for constructing temperature boundary conditions around $\omega$. These evaluations correspond to either a vertex of the domain $\omega$ or a point on the boundary between neighboring coarse-scale elements and are calculated at a $t^{n}$ as shown in Figure 2b.

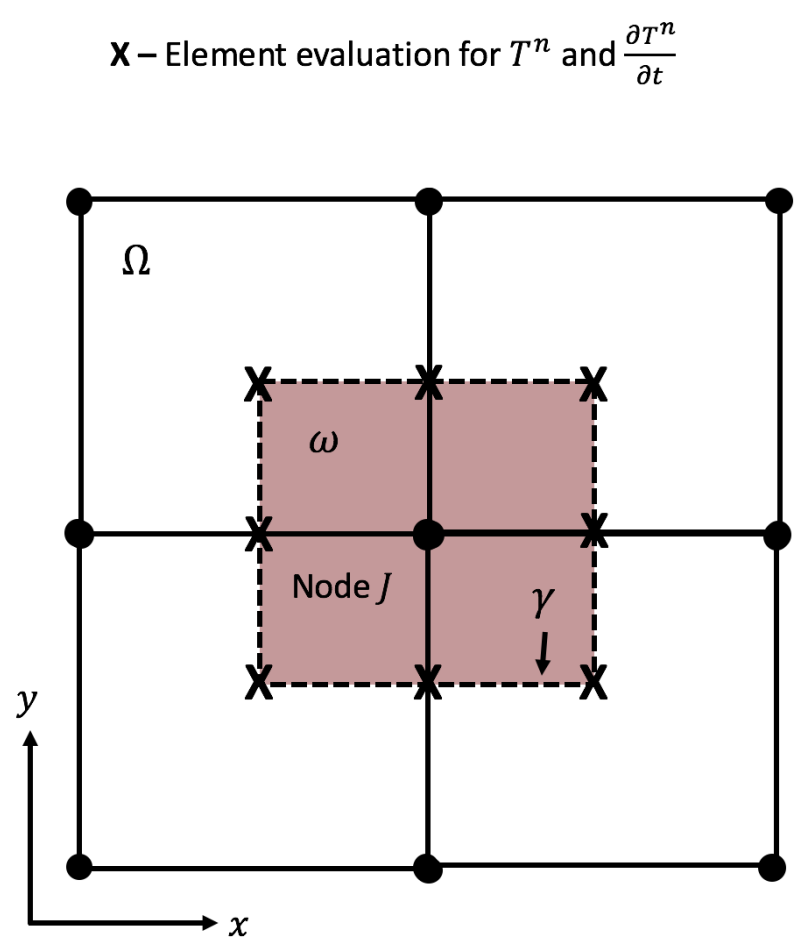

(a)

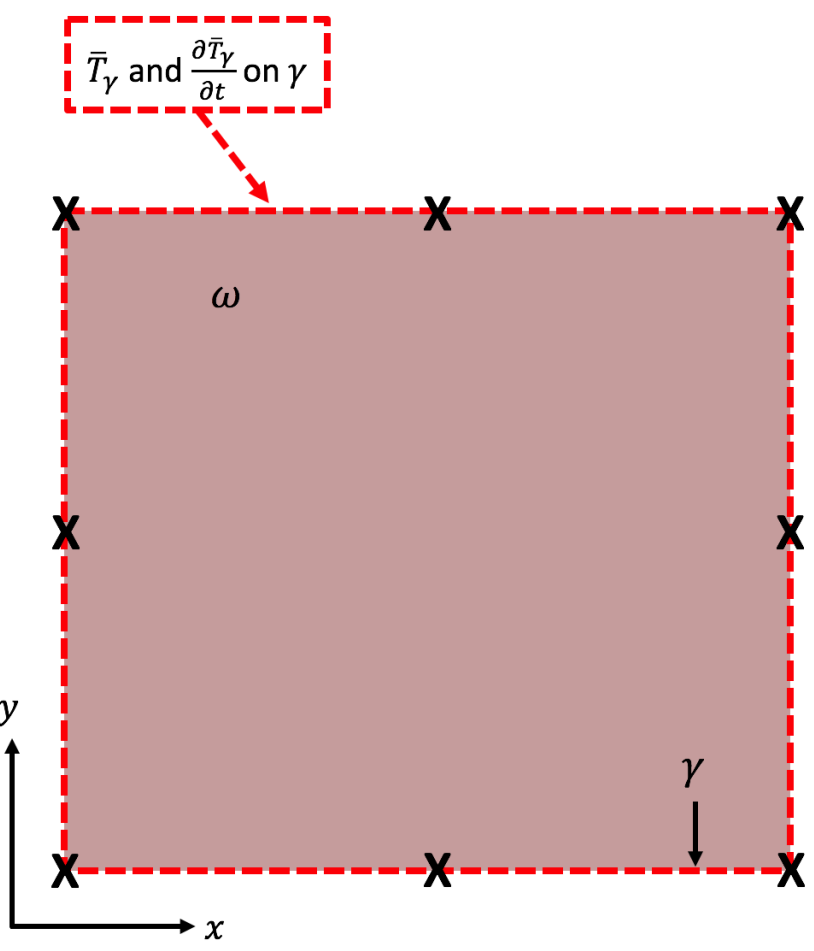

(b)

Figure 2: (a) Element patch in $\Omega$ showing 8 coarse-scale evaluation points used to evaluate fine-scale Dirichlet boundary conditions and (b) edges and vertices on $\gamma$ corresponding to the coarse-scale evaluation points.

At each evaluation point the temperature $T^{n}$ and its rate $\frac{\partial T^{n}}{\partial t}$ will be calculated. From these evaluation points, a full inter-element piecewise linear temperature and temperature rate field, denoted as $\bar{T}_{\gamma}$ and $\frac{\partial \bar{T}_{\gamma}}{\partial t}$, respectively, are constructed along the boundaries $\gamma$. These two fields are used to construct a time-dependent Dirichlet boundary condition applied along $\gamma$ to evolve $\omega$ over a coarse-scale time interval $\left[t^{n}, t^{n+1}\right]$. The Dirichlet boundary condition enforced at some time $t^{k}$ during the fine-scale simulation, with $t^{k} \in\left[t^{n}, t^{n+1}\right]$, is evaluated as

$$
\theta=\bar{T}_{\gamma}+\int_{t^{n}}^{t^{k}} \frac{\partial \bar{T}_{\gamma}}{\partial t} d t \text { on } \gamma
$$




\subsubsection{Results for Concurrent Coupling through Temperature and Flux Boundary Conditions}

A series of numerical tests with various sizes of the fine scale domain $\omega$ are performed to examine the energy conservation between $\Omega$ and $\omega$ when utilizing both the temperature and flux boundary conditions to link the two scales. Figure 3 shows the dimensions and boundary conditions applied on the coarse scale. Neumann boundary conditions are applied on all four edges, the coarse-scale node of interest is located at $(0.24,0.5)$. The values of $\kappa, \rho$ and $c_{p}$ correspond to $\kappa_{0}, \rho_{0}$ and $c_{p o}$ in Table 1, respectively. Both the coarse scale and fine scale have a square geometry, with the coarse scale having a side length of 1 . No source term $S$ is applied over $\Omega$.

All of the tests are run to a final time of $t=1.0$. We measure each method's ability to conserve energy by computing the nodal temperature error $E_{J}$ using Eq. (31) at every coarse scale time step. The mean error $E_{\text {mean }}$ is computed over time to measure energy conservation between scales. Table 2 shows the resulting mean energy errors over time for varying side lengths of $\omega$. As seen from Table 2, the mean error $E_{\text {mean }}$ for our proposed method varies between $10^{-12}$ and $10^{-13}$ (close to machine accuracy), regardless of the size of the fine scale domain $\omega$. The mean error is much higher when using a temperature boundary condition (on the order of $10^{-4}$ ). This indicates that energy is not as accurately conserved between the scales $\Omega$ and $\omega$ when enforcing temperature or Dirichlet boundary conditions on $\omega$. Furthermore, Table 2 shows that the mean error increases when the size of $\omega$ increases when utilizing temperature boundary conditions, indicating an approximately first order dependence on the length scale of the fine scale domain $\omega$.

Table 2: Energy results for Problem 1

\begin{tabular}{|l|l|l|}
\hline Side Length for $\omega$ & $E_{\text {mean }}$ for Temperature BC & $E_{\text {mean }}$ for Proposed Method \\
\hline $5.0 \times 10^{-4}$ & $5.22 \times 10^{-5}$ & $6.5866 \times 10^{-12}$ \\
$1.0 \times 10^{-3}$ & $9.8353 \times 10^{-5}$ & $4.4249 \times 10^{-12}$ \\
$2.5 \times 10^{-3}$ & $2.1670 \times 10^{-4}$ & $6.0697 \times 10^{-13}$ \\
$5.0 \times 10^{-3}$ & $3.7443 \times 10^{-4}$ & $6.2014 \times 10^{-13}$ \\
$1.0 \times 10^{-2}$ & $6.2976 \times 10^{-4}$ & $2.3038 \times 10^{-13}$ \\
\hline
\end{tabular}

\subsection{Simple Heat Transfer Problem in 2D}

Figure 3 shows the configuration and boundary conditions for Problem 2. Discretizations of $100 \times 100$ and $20 \times 20$ elements are used for the coarse and fine scale, respectively. The fine scale domain $\omega$ is constructed around a node within $\Omega$ with coordinates $(0.24,0.5)$. The coarse-scale time step is $\Delta \bar{t}=5.0 \times 10^{-5}$ and the fine-scale time step is $\Delta t=1.25 \times 10^{-9}$. Material properties on $\omega$ are identical to those of $\Omega$ with $\overline{\boldsymbol{K}}=\kappa \boldsymbol{I}$ where $\boldsymbol{I}$ is the identity tensor. Fluxes are applied at each of the four boundaries, with their respective values shown in Figure 3.

A time-dependent volumetric heat source, with a Gaussian profile in space, is applied throughout $\Omega$ and calculated as 


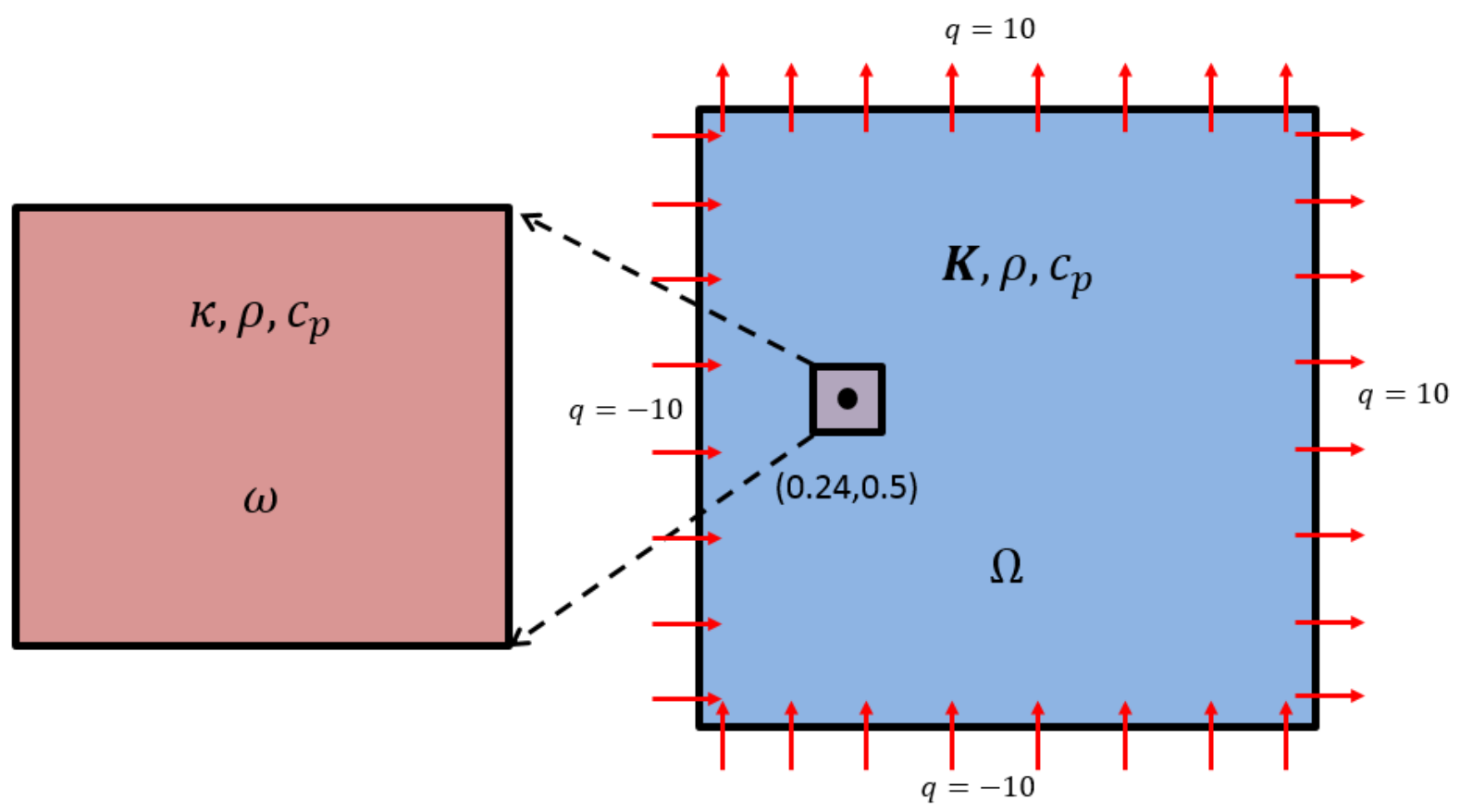

Figure 3: Homogeneous heat transfer configuration for Problems 1 and 2. Both $\Omega$ and $\omega$ have homogeneous and isotropic material properties. $\Omega$ is a unit square with a fixed length of 1.0 and $\omega$ is a unit square with a variable length in Problem 1 and a fixed length of $1.0 \times 10^{-3}$ in Problem 2

$$
S=A \exp \left(\frac{-\left|\boldsymbol{x}-\boldsymbol{x}_{s}\right|^{2}}{2 \sigma^{2}}\right) \cos (\lambda t)
$$

where $A$ determines the strength of the heat source, $\sigma$ controls the spread of the source term profile in both the $x$ and $y$ direction, $\boldsymbol{x}_{s}$ is the position of the center of the Gaussian distribution, and $\lambda$ determines the frequency of the source term. This time dependent source term is applied to Problems 2 and 3 with parameters listed in Table 3.

Table 3: Source term parameters used for Problems 2 and 3

\begin{tabular}{|c|c|c|}
\hline Parameter & Problem 2 & Problem 3 \\
\hline$A$ & 150 & 150 \\
$\sigma$ & 0.5 & 0.5 \\
$\lambda$ & 50 & 250 \\
\hline
\end{tabular}

This numerical example was run to a final time of $t=2.0$. The temperature history at the node of interest is shown in Figure 4, indicating a rapidly changing temperature. As in Problem 1, the absolute error between the nodal temperatures when running with and without an embedded fine scale region is tracked throughout the duration of each simulation and defined in Eq. (31) as $E_{J}$. The mean error throughout the duration of this simulation is $E_{\text {mean }}=1.4402 \times 10^{-11}$, with a minimum of $3.0293 \times 10^{-15}$ and maximum of $2.8383 \times 10^{-11}$. These errors are on the order of machine accuracy, and demonstrate that the rate of energy throughout $\omega$ is consistent with that of the node of interest. 


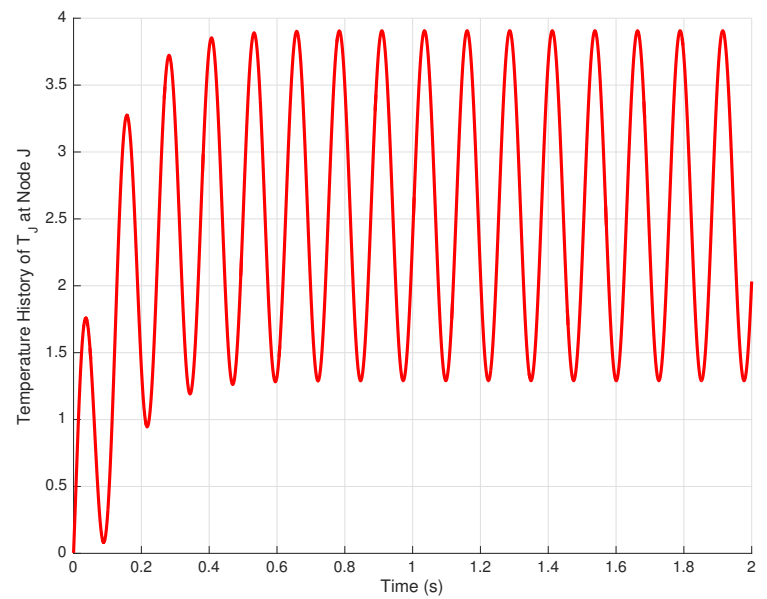

Figure 4: Temperature history for Problem 1

417

\subsection{Heat Transfer With Composite Microstructure}

Figure 5 shows the boundary conditions and configuration for the Problem 3. The domain sizes and shapes are identical to the ones used for Problem 2. In contrast to Problem 2, the domain $\omega$ in Problem 3 is assumed to have a repeating layered composite microstructure with alternating material properties. Figure 5 shows the periodic microstructure of the domain $\omega$. The coarsescale time step is $\Delta \bar{t}=5.0 \times 10^{-7}$ and the fine-scale time step is $\Delta t=1.25 \times 10^{-11}$. The material parameters for this example are listed in Table 1 . The material properties of the periodic microstructure are homogenized to yield an effective heat capacitance $\bar{\rho} \overline{c_{p}}$ and thermal conductivity $\overline{\boldsymbol{K}}$ at each Gauss integration point within the coarse scale $\Omega$. 


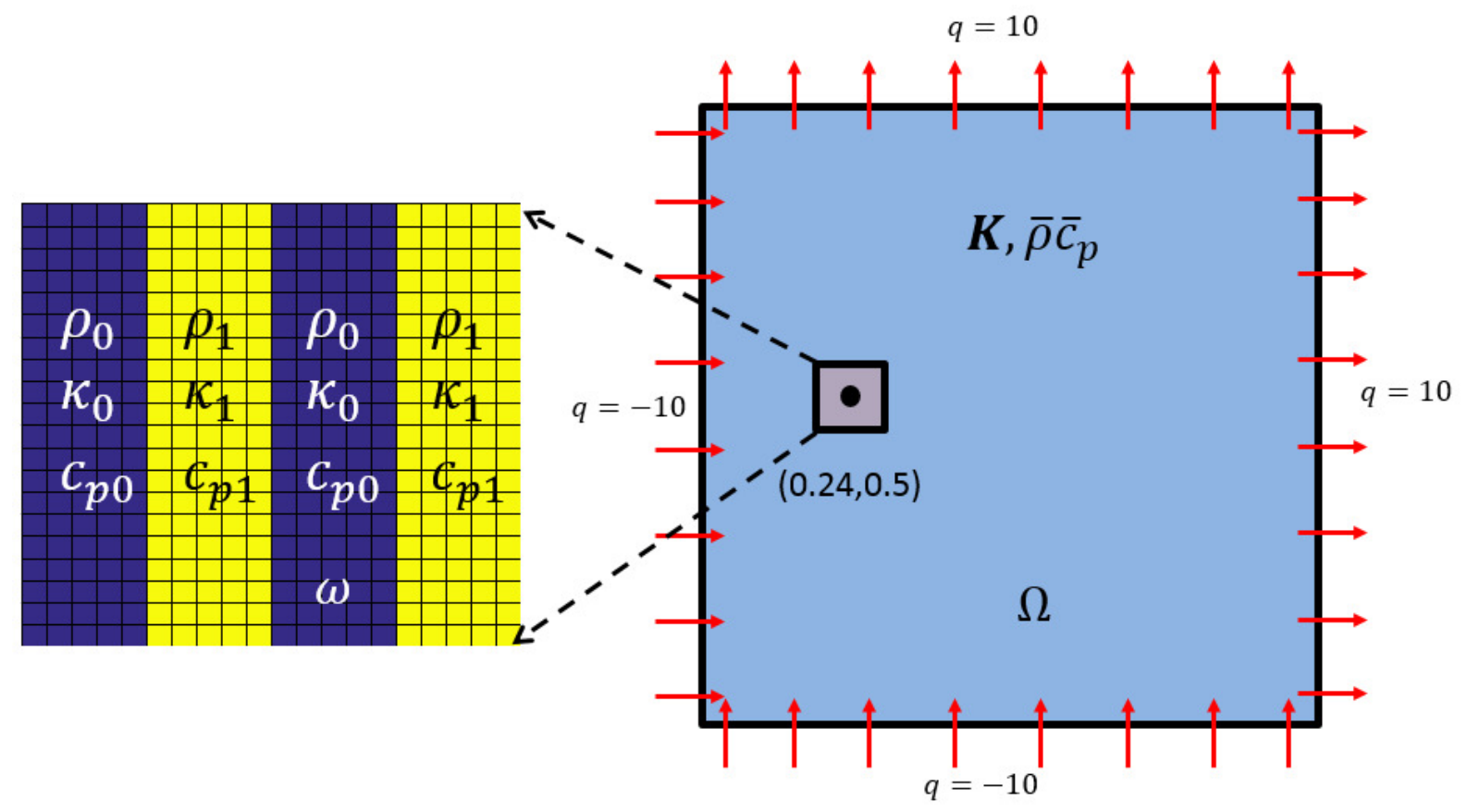

Figure 5: Composite microstructure configuration for Problem 3. A lamellar composite microstructure is introduced for spatially varying material properties in $\omega$. An effective conductivity tensor $\overline{\boldsymbol{K}}$ and capacitance $\bar{\rho} \bar{c}_{p}$ is calculated form homogenizing the properties in $\omega . \Omega$ and $\omega$ are unit squares with fixed lengths of 1.0 and $1.0 \times 10^{-3}$, respectively

\subsubsection{Results Of Composite Test}

This numerical example was also run to a final time of $t=0.2$. The temperature history at the node of interest is shown in Figure 6, and again indicates a rapidly changing nodal temperature in time. The mean error $E_{J}$ throughout the duration of this simulation is $E_{\text {mean }}=2.8042 \times 10^{-11}$, with a minimum and maximum of $5.2042 \times 10^{-18}$ and $8.1960 \times 10^{-12}$, respectively.

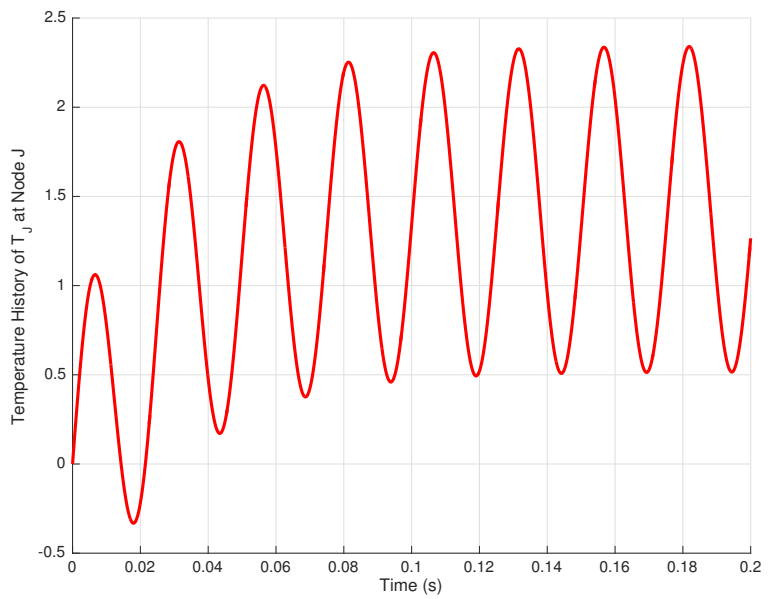

Figure 6: Temperature history for Problem 3 


\subsection{Heat Transfer with Phase Transformation}

Solidification occurs in a diverse range of industrial processes such as metal processing, casting solidification, and additive manufacturing $[42,43]$. This process is characterized by the formation of distinct phases within a system with a boundary separating these two phases, introducing a moving boundary problem for modeling the process. Numerical treatments and methods have been developed for computationally handling these phase transformations at a variety of scales.

For the numerical example considered here, a phase field model is implemented on the fine scale $\omega$ to simulate a solidification process. Phase field models have been widely used in applications that incorporate solidification dynamics, and belong to a class of methods that implicitly track the interface between two phases through solving an additional partial differential equation that evolves an auxiliary field known as the phase field order parameter $\phi$. This order parameter indicates the bulk phase of a material, with $\phi=0$ for one phase and $\phi=1$ for the other. The interface is represented as a smooth continuous changes between $\phi=0$ and 1 . The following section gives a brief overview of the phase field model implemented in this paper. For more details, the reader should refer to text given by Provatas and Elder [44].

\subsubsection{Development of the Fine-scale Phase Field Model for Solidification}

The derivation of the phase field evolution equations begin with a definition of the total free energy functional $F[\phi, \theta]$ defined over an arbitrary volume $V$.

$$
F(\phi, \theta)=\int_{V}\left(\frac{1}{2}|\epsilon(\hat{\boldsymbol{n}}) \nabla \phi|^{2}+f(\phi, \theta)\right) d V
$$

where $f(\phi, \theta)$ represents a free energy density function, dependent on both the phase field $\phi$ and temperature $\theta$, and $\epsilon(\hat{\boldsymbol{n}})$ is a gradient energy coefficient which controls the width of the diffuse interface. In equation 34 , we have written this coefficient as $\epsilon(\hat{\boldsymbol{n}})$ to indicate dependence on the normal $\hat{\boldsymbol{n}}$ of the interface, ultimately introducing anisotropy. For the following example we will only focus on isotropic solidification, making $\epsilon(\hat{\boldsymbol{n}})$ a constant $\epsilon$ independent of $\hat{\boldsymbol{n}}$. The free energy density, $f(\phi, \theta)$, is composed of the sum of two terms: $f(\phi, \theta)=g(\phi)+\frac{L\left(\theta-\theta_{m}\right)}{H \theta_{m}} h(\phi)$. The temperatureindependent term $g(\phi)$ is a double-well energy potential, and $h(\phi)$ is an interpolating function that accounts for thermal effects in $f(\phi, \theta)$. The free energy density $f(\phi, T)$ can be formally written as

$$
\begin{aligned}
g(\phi) & =\phi^{2}(1-\phi)^{2} \\
h(\phi) & =(3-2 \phi) \phi^{2} \\
f(\phi, T) & =\phi^{2}(1-\phi)^{2}+\frac{L\left(\theta-\theta_{m}\right)}{H \theta_{m}}(3-2 \phi) \phi^{2}
\end{aligned}
$$


Here, $L$ is a latent heat term, $H$ acts as an activation barrier, and $\theta_{m}$ is the melting temperature. The solid and liquid phases are represented as $\phi=0$ and $\phi=1$, respectively.

The phase field evolution equation is formulated by taking a variational derivative of the free energy functional $F(\phi, \theta)$. For solidification, the phase field order parameter is a non-conserved variable and evolves to minimize the total free energy within a system. The evolution equation for non-conserved order parameters are formulated through the Allen-Cahn equation, defined as

$$
\frac{\partial \phi}{\partial t}=-M \frac{\delta F}{\delta \phi}=-M\left[\epsilon^{2} \nabla^{2} \phi-\frac{\partial f}{\partial \phi}\right]
$$

where $M$ is a mobility parameter. Using the definition of the free energy density in Eq. (35c), the evolution of the phase field is given by

$$
\tau \frac{\partial \phi}{\partial t}=\nabla \cdot\left(\epsilon^{2} \nabla \phi\right)-2\left(\phi-3 \phi^{2}+2 \phi^{3}\right)-\frac{6 L}{H T_{m}}\left(\theta-\theta_{m}\right) \phi(1-\phi)
$$

Here, the parameter $M$ has been absorbed into $\tau$, which gives a relaxation time scale for the phase field variable.

\subsubsection{Governing Equations at the Fine Scale}

For the numerical example considered here, Eq. (37) is subjected to zero-flux boundary conditions for $\phi$. Additionally, the heat equation posed in Eq. (10a) is modified to contain a latent effect due to the phase field [44]. The strong form equations acting over the fine scale $\omega$ are then

$$
\begin{array}{r}
\nabla \cdot \kappa \nabla \theta+s+L \frac{\partial h}{\partial \phi} \frac{\partial \phi}{\partial t}=\rho c_{p} \frac{\partial \theta}{\partial t} \quad \text { in } \omega \\
\nabla \cdot\left(\epsilon^{2} \nabla \phi\right)-2\left(\phi-3 \phi^{2}+2 \phi^{3}\right)-\frac{6 L}{H T_{m}}\left(\theta-\theta_{m}\right) \phi(1-\phi)=\tau \frac{\partial \phi}{\partial t} \quad \text { in } \omega \\
-\boldsymbol{q} \cdot \boldsymbol{n}=q \quad \text { on } \gamma_{N}
\end{array}
$$

The additional latent heat term in Eq. (38a) acts as a source that absorbs energy during a phase transformation without any change in temperature, and $\boldsymbol{q}$ is calculated from Eq. (24).

\subsubsection{Coarse-scale Approximation using the Apparent Heat Capacity Method}

The coarse scale will utilize the apparent heat capacity (AHC) method to model the solidification process. This method, originally introduced by Hashemi and Sliepcevich [45], approximates phase transitions within a finite temperature range $T_{S}<T<T_{L}$. Within this range, the heat capacity is increased to account for energy dissipation or absorption through the latent heat. The solidus and liquidus temperatures of a material are denoted as parameters $T_{S}$ and $T_{L}$, respectively. In our 
example, it is assumed that the latent heat is released uniformly in the phase change temperature range. With this assumption, the effective specific heat capacity $c_{p}$ at a node $I$ with a temperature of $T_{I}^{n}$ at some temporal state $n$ is then

$$
c_{p}\left(T_{I}^{n}\right)= \begin{cases}c_{p s}, & T_{I}^{n}<T_{S} \\ c_{p}+\frac{L}{T_{L}-T_{S}}, & T_{S} \leq T_{I}^{n} \leq T_{L} \\ c_{p l}, & T_{L}<T_{I}^{n}\end{cases}
$$

where $c_{p s}$ and $c_{p l}$ are the specific heat capacity of the solid and liquid phase, respectively. In the following numerical example, we assume $c_{p s}=c_{p l}=c_{p}$.

\subsubsection{Results of Solidification Test}

Figure 7 shows the problem definition and configuration for our numerical example simulating solidification. The domains $\Omega$ and $\omega$ are unit squares with lengths $5.0 \times 10^{0}$ and $1.0 \times 10^{-3}$, respectively. The coarse scale is discretized with $150 \times 150$ elements and the fine scale is discretized with $40 \times 40$. The domain $\omega$ is constructed around a node within $\Omega$ located at coordinates $(0.033,0.133)$. Additionally, an outflow flux is applied on the left edge of $\Omega$. No external source terms are considered for this example.

A composite microstructure with varying thermal conductivity is prescribed in $\omega$ and this unit cell is assumed to repeat periodically throughout the coarse scale $\Omega$. The thermal conductivity of the periodic microstructure in $\omega$ is homogenized using the method outlined in section 2.3.4 to give an effective coarse-scale thermal conductivity tensor $\boldsymbol{K}$ at each Gauss integration point within the coarse scale $\Omega$. The coarse-scale time step is $\Delta \bar{t}=1.0 \times 10^{-7}$ and the fine-scale time step is $\Delta t=6.25 \times 10^{-11}$. The material parameters used for this example are given in Table 1 with $\rho=\rho_{0}$ and $c_{p}=c_{p o}$. The phase field and AHC parameters are listed in Table 4. For this example, the simulation is run until the fine scale domain has fully solidified, which is approximately at $t=8 \times 10^{-4}$ seconds.

Table 4: Thermodynamic phase field parameters for Problem 4

\begin{tabular}{|c|c|}
\hline Parameter & Value \\
\hline$L$ & 10.0 \\
$T_{L}$ & 1.033 \\
$T_{m}$ & 1.0 \\
$T_{S}$ & 0.966 \\
$H$ & 0.1 \\
$\varepsilon$ & $5.0 \times 10^{-5}$ \\
$\tau$ & $8.5 \times 10^{-5}$ \\
\hline
\end{tabular}


Figure 8 shows the temperature history at the node of interest for both simulations. From the figure, it can be seen that there is a difference in the predicted energy dissipation between fine scale phase field model and AHC method during solidification. The resulting temperature histories of the two simulations are expected to differ, as the AHC is only a coarse scale approximation for solidification that is sensitive to the assumed temperature interval for phase transition whereas the phase field model provides a highly resolved depiction of the phase transition.

In addition to resolving a different energy dissipation at the node of interest, the concurrently coupled phase field model evolved over the fine scale $\omega$ is able to provide a spatially varying phase composition over time. Figure 9 shows "snapshots" of these phase compositions throughout the solidification process. Figures 9a, 9b, 9c and 9d correspond to the temperature points marked "a", "b", "c" and "d" in Figure 8

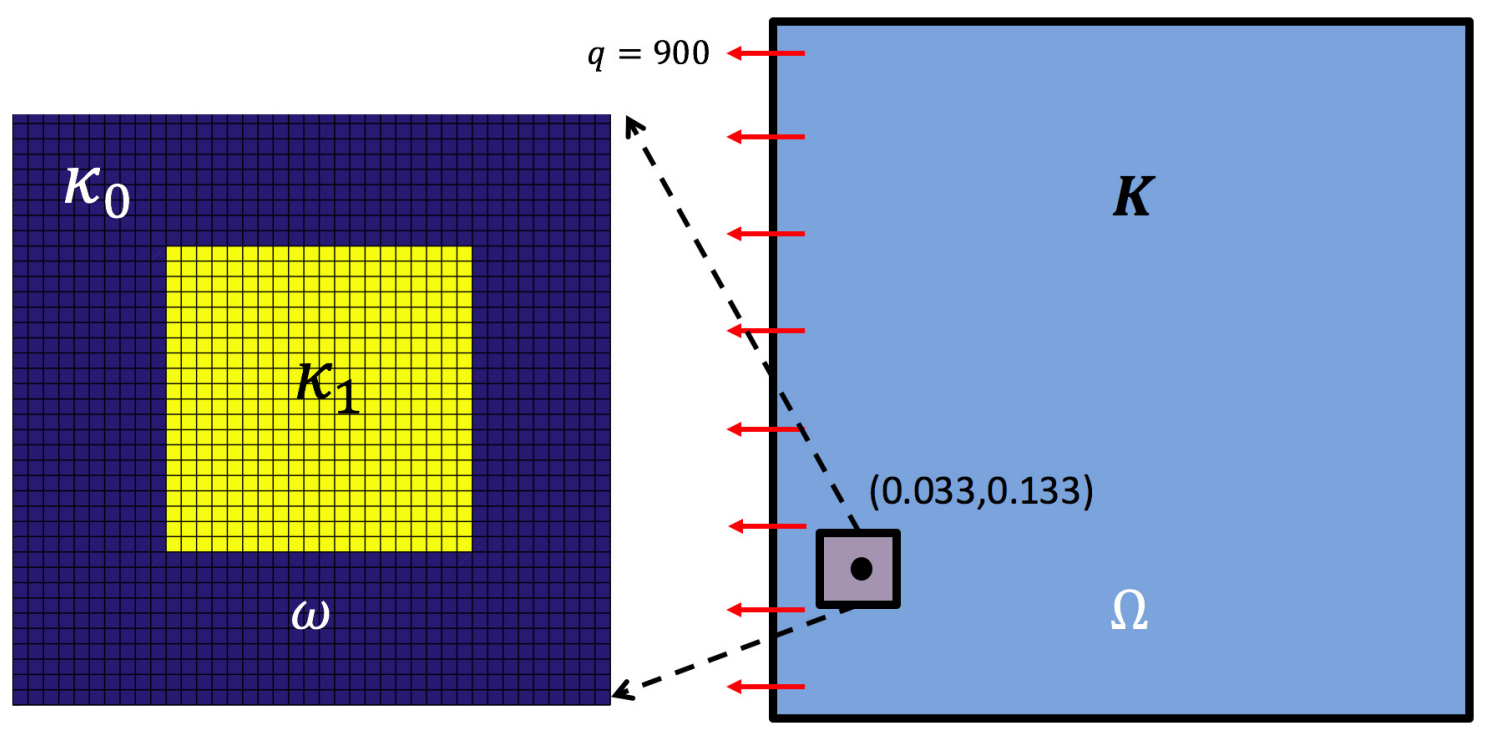

Figure 7: Composite microstructure configuration for Problem 4 introducing a spatially varying thermal conductivity $\kappa$ in $\omega$. An effective conductivity tensor $\overline{\boldsymbol{K}}$ is calculated from homogenizing the conductivity in $\omega . \Omega$ and $\omega$ are unit squares with fixed lengths of 5.0 and $1.0 \times 10^{-3}$, respectively

\subsection{Heat Transfer With Composite Microstructure in 3D}

The last numerical example is conducted in a 3D setting. The coarse-scale problem is shown in Figure 10b. The domains $\Omega$ and $\omega$ are unit cubes with lengths $1.0 \times 10^{0}$ and $1.0 \times 10^{-3}$, respectively. The fine scale is constructed around a node in $\Omega$ located at $(0.1,0.15,0.05)$. Outflow fluxes are applied to 3 boundaries shown in Figure 10b. No volumetric source term is considered here.

The coarse scale has a repeating composite microstructure with differing thermal conductivities. Figure 10a shows the domain $\omega$ with the corresponding periodic microstructure. The periodic microstructure is homogenized for an effective thermal conductivity tensor $\overline{\boldsymbol{K}}^{\text {eff }}$ at each Gauss integration point in the domain $\Omega$. The coarse-scale time step is $\Delta \bar{t}=1.25 \times 10^{-4}$ and the fine-scale time step is $\Delta t=5.0 \times 10^{-10}$. 


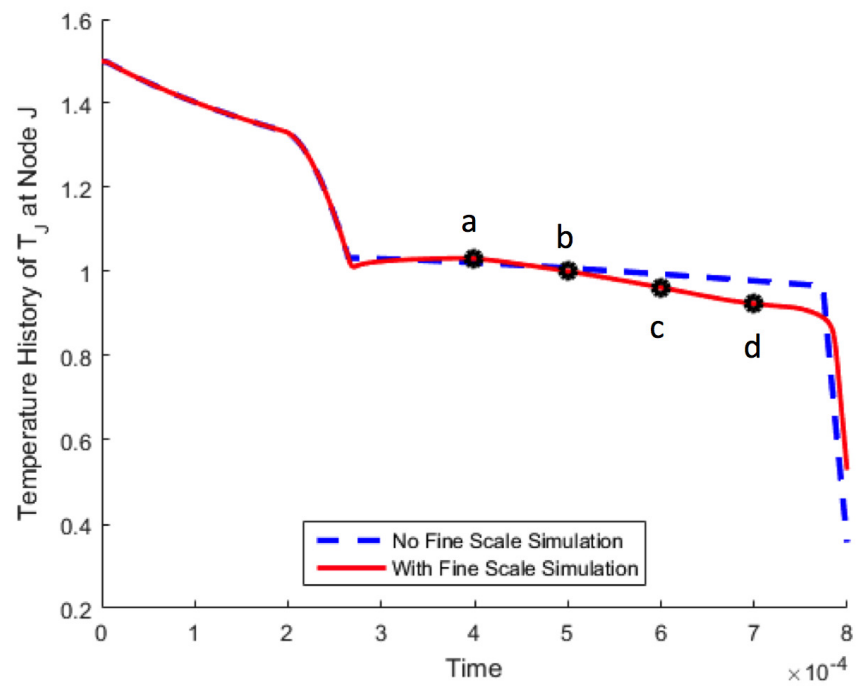

Figure 8: Temperature history for Problem 4 with and without a concurrently coupled fine-scale simulation

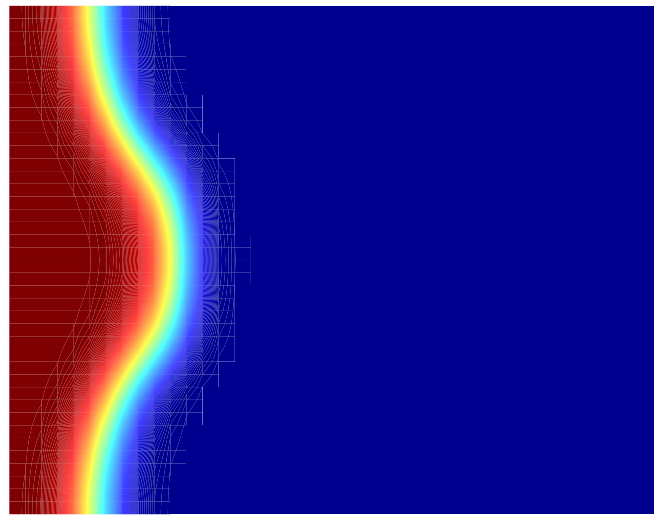

(a) Phase field distribution at point "a"

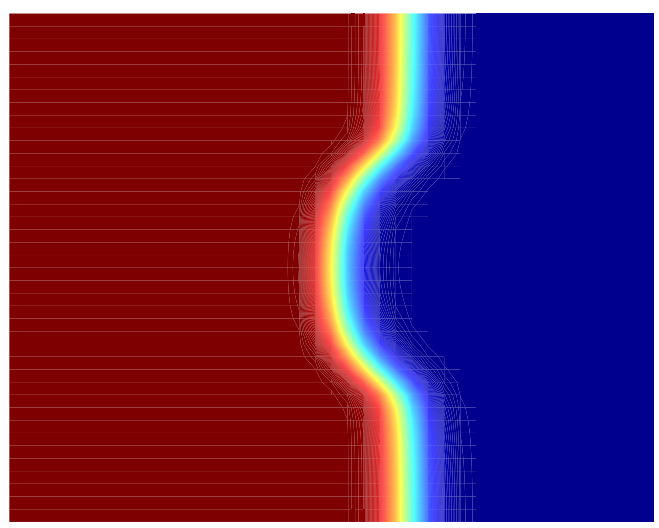

(c) Phase field distribution at point "c"

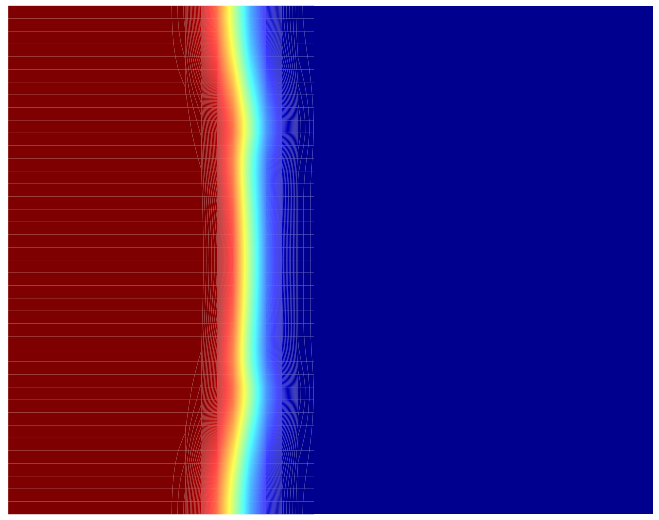

(b) Phase field distribution at point "b"

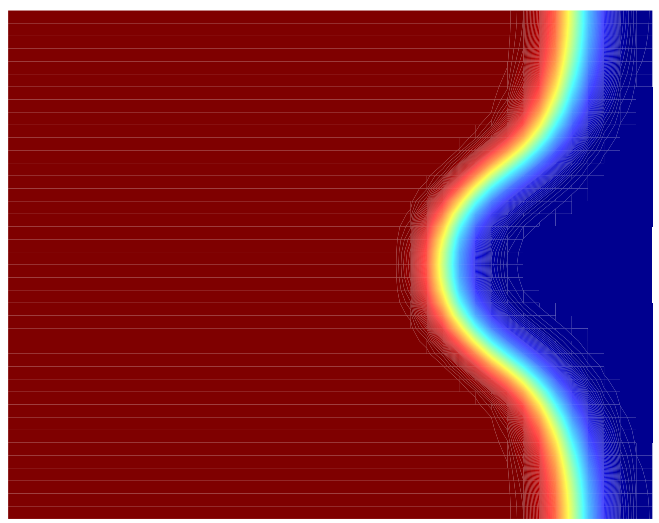

(d) Phase field distribution at point "d"

Figure 9: Phase field distributions corresponding to select points indicated on Figure 8 


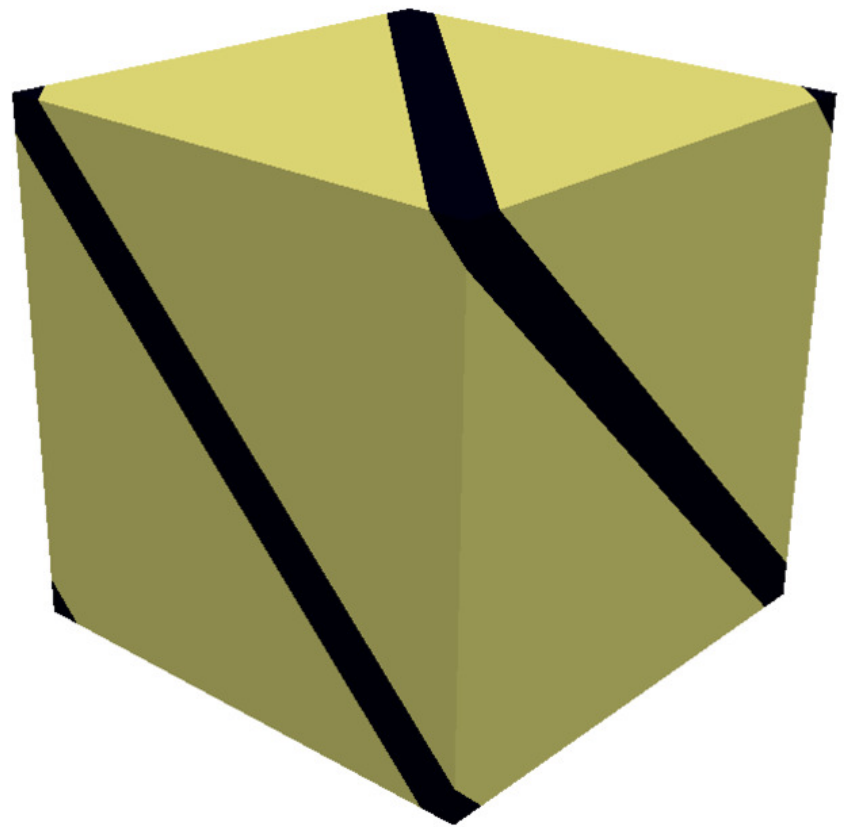

(a)

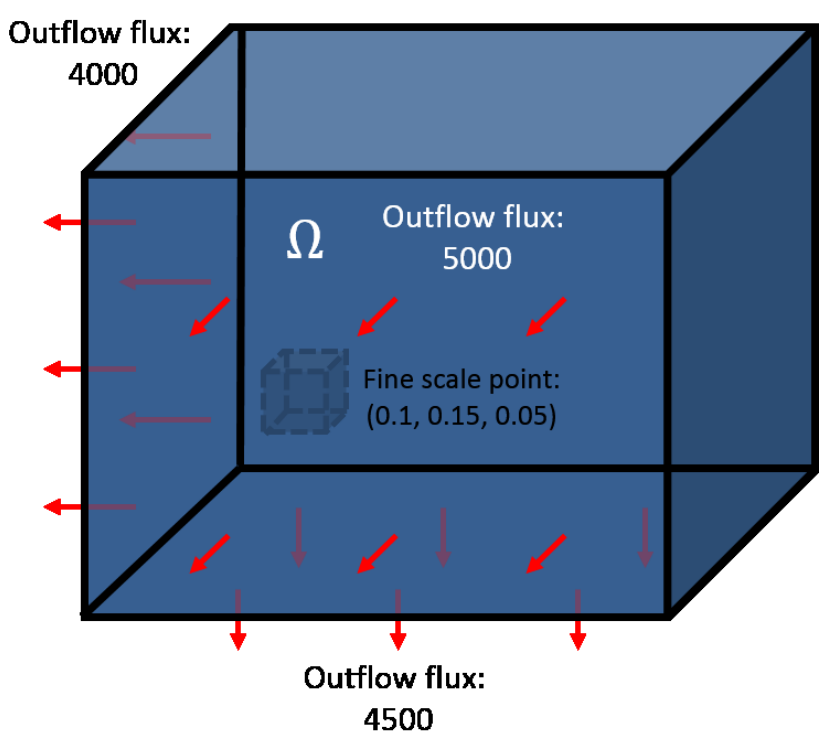

(b)

Figure 10: 3D heat transfer configuration for Problem 5. (a) A composite microstructure is introduced for spatially varying material properties in $\omega$. (b) Problem definition for the coarse-scale problem $\Omega$ that contains the microstructure $\omega$. An effective conductivity tensor $\overline{\boldsymbol{K}}$ is calculated from homogenizing the properties in $\omega$. $\Omega$ and $\omega$ are unit cubes with fixed lengths of $1.0 \times 10^{0}$ and $1.0 \times 10^{-3}$, respectively

This numerical example was run until $t=1.5$. The temperature history at the node of interest is shown in Figure 11. The mean error $E_{J}$ throughout the duration of the simulation is $3.8842 \times 10^{-9}$, with a maximum of $1.3562 \times 10^{-8}$ and minimum of $5.5821 \times 10^{-16}$.

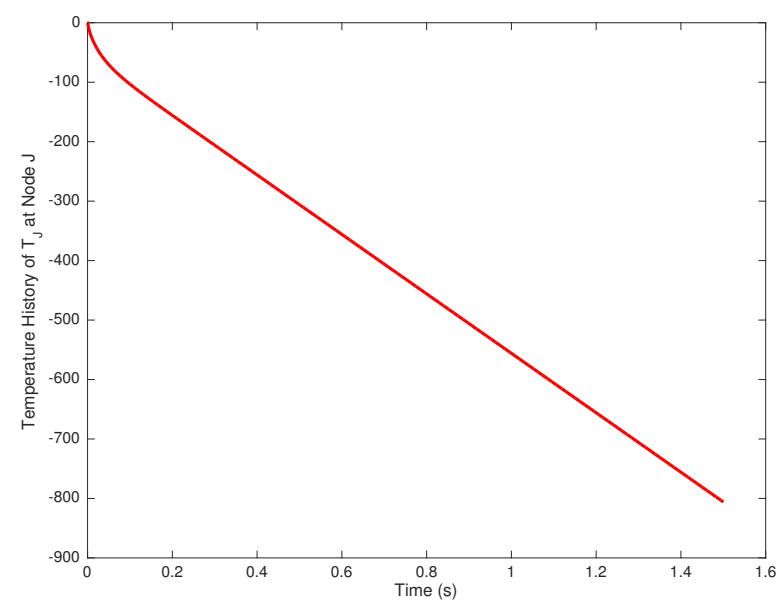

Figure 11: Temperature history for Problem 5 


\section{Conclusion}

We have presented a method for coupling multilevel finite element models into a single concurrent multiscale simulation. A fine scale discretization resolving any spatial complexities is linked to a specified nodal point within the coarse scale. The solution at the specified coarse scale nodal point is used to construct a flux field within the fine scale. This flux field is then used to construct Neumann boundary conditions on the fine scale boundaries to ensure a consistent energetic evolution between the fine scale and the coarse scale node. Results from the fine scale are then homogenized through an energy-based scheme.

Numerical examples are run to demonstrate the capabilities of our proposed method. Section 3.1 illustrates the accuracy in energy conservation between scales when using Neumann in place of Dirichlet boundary conditions. Examples in Section 3.2 and 3.3 show our proposed method's ability to handle a rapidly-changing temperature field and a repeating composite microstructure within the coarse scale domain. A solidification example is performed in Section 3.4 to showcase our proposed method's advantages; a detailed temperature-phase field coupled numerical model is implemented on the fine scale to spatially resolve the solidification profile of a composite microstructure. The results of this fine scale computation are homogenized back to the coarse scale to predict a temperature profile that differs from traditional approximations for solidification. Lastly, a 3D example with a spatially varying composite microstructure is performed in Section 3.5 to extend our proposed method to a 3D setting. Our proposed method does not follow traditional computational homogenization approaches; the fine scale domain is only linked to a small set of critical regions within the coarse scale where a high resolution is necessary. As a result, we do not use the average microscopic heat flux as the coarse-scale flux at every integration point as done in typical computational homogenization or $\mathrm{FE}^{2}$ methods. Instead, we see our method as complementary to these methods: at typical coarse scale integration points, an analytical or computational homogenization technique could be used if desired to obtain an average flux. For our aforementioned examples, we considered a linearized formulation for the thermal flux and utilized a homogenization approach outlined in Section 2.3.4 for evaluating a semi-analytical effective coarse-scale conductivity to resolve heterogeneous microstructures. We emphasize that this method of homogenization is not a primary focus of our proposed concurrent method, and for general nonlinear cases a different homogenization approach, such as $\mathrm{FE} 2^{2}$, could easily be used in conjunction with our approach.

In this paper, we have only performed simulations where two scales were concurrently coupled. It should be noted, however, that our proposed method can handle multiple nested scales. The only limiting factors in a concurrently coupling nested scales with our proposed method are computational resources and time available for simulation. We expect that the majority of the computational costs will be during the evolution of the fine-scale system. However, it should be noted that our proposed concurrent method has been targeted at modeling phase transition applications. For these 
types of applications, we are able to determine the onset of phase transition based on the state of the system (e.g. if the temperature is in the vicinity of the liquidus/solidus points). Therefore, computational expenses can be minimized by activating the fine-scale coupling over specific time periods such that we only capture the phase transition with a high resolution at the fine scale. Lastly, the only information necessary for concurrently coupling scales using our proposed method is the solution at the node of interest; from this the appropriate boundary conditions at the subsequent scales are constructed to evolve the system of equations. Therefore, parallelization of the proposed method is a straightforward task, as both coarse and fine scales can be independently parallelized.

The phase morphology of raw materials undergoing manufacturing processes are sensitive to the operating conditions. Our proposed method can elucidate the driving physics that drive these phase transitions and ultimately establish a link between processing parameters and the final phase morphology. A specific application that may benefit greatly from this method is additive manufacturing (AM), where cyclic heating and cooling cycles throughout the process have drastic effects on the final microstructure morphology e.g. grain size and orientation. Establishing this link between processing parameters and the final as-built microstructure can lead to the design of new materials.

\section{Acknowledgments}

This work is supported by National Institute of Standards and Technology (NIST) and Center for Hierarchical Materials Design (CHiMaD) under grant No. 70NANB13H194 and 70NANB14H012. This material is based upon work supported by the National Science Foundation Graduate Research Fellowship under Grant No. DGE-1324585.

\section{References}

[1] J. Fish, Practical multiscaling, John Wiley \& Sons, 2013.

[2] V. Shenoy, R. Miller, E. Tadmor, D. Rodney, R. Phillips, M. Ortiz, An adaptive finite element approach to atomic-scale mechanicsthe quasicontinuum method, Journal of the Mechanics and Physics of Solids 47 (1999) 611-642.

[3] G. J. Wagner, W. K. Liu, Coupling of atomistic and continuum simulations using a bridging scale decomposition, Journal of Computational Physics 190 (2003) 249-274.

[4] S. Xiao, T. Belytschko, A bridging domain method for coupling continua with molecular dynamics, Computer Methods in Applied Mechanics and Engineering 193 (2004) 1645-1669. 
[5] W. Chen, J. Fish, A mathematical homogenization perspective of virial stress, International Journal for Numerical Methods in Engineering 67 (2006) 189-207.

[6] W. Chen, J. Fish, A generalized space-time mathematical homogenization theory for bridging atomistic and continuum scales, International Journal for Numerical Methods in Engineering 67 (2006) 253-271.

[7] J. Fish, W. Chen, R. Li, Generalized mathematical homogenization of atomistic media at finite temperatures in three dimensions, Computer Methods in Applied Mechanics and Engineering 196 (2007) 908-922.

[8] Y. Lee, C. Basaran, A multiscale modeling technique for bridging molecular dynamics with finite element method, Journal of Computational Physics 253 (2013) 64-85.

[9] R. E. Miller, E. B. Tadmor, A unified framework and performance benchmark of fourteen multiscale atomistic/continuum coupling methods, Modelling and Simulation in Materials Science and Engineering 17 (2009) 053001.

[10] A. Brandt, Multi-level adaptive solutions to boundary-value problems, Mathematics of Computation 31 (1977) 333-390.

[11] W. L. Briggs, S. F. McCormick, et al., A multigrid tutorial, SIAM, 2000.

[12] J. Fish, V. Belsky, Multigrid method for periodic heterogeneous media part 1: Convergence studies for one-dimensional case, Computer Methods in Applied Mechanics and Engineering 126 (1995) 1-16.

[13] J. Fish, V. Belsky, Multi-grid method for periodic heterogeneous media part 2: Multiscale modeling and quality control in multidimensional case, Computer Methods in Applied Mechanics and Engineering 126 (1995) 17-38.

[14] H. Boffy, C. H. Venner, Multigrid solution of the 3D stress field in strongly heterogeneous materials, Tribology international 74 (2014) 121-129.

[15] W. Cecot, M. Oleksy, High order FEM for multigrid homogenization, Computers \& Mathematics with Applications 70 (2015) 1391-1400.

[16] T. Y. Hou, X.-H. Wu, A multiscale finite element method for elliptic problems in composite materials and porous media, Journal of Computational Physics 134 (1997) 169-189.

[17] G. Allaire, R. Brizzi, A multiscale finite element method for numerical homogenization, Multiscale Modeling \& Simulation 4 (2005) 790-812.

[18] S. Soghrati, I. Stanciulescu, Systematic construction of higher order bases for the finite element analysis of multiscale elliptic problems, Mechanics Research Communications 52 (2013) 11-18. 
[19] T. J. Hughes, G. R. Feijóo, L. Mazzei, J.-B. Quincy, The variational multiscale method a paradigm for computational mechanics, Computer Methods in Applied Mechanics and Engineering 166 (1998) 3-24.

[20] T. J. Hughes, L. Mazzei, K. E. Jansen, Large eddy simulation and the variational multiscale method, Computing and Visualization in Science 3 (2000) 47-59.

[21] V. John, S. Kaya, A finite element variational multiscale method for the navier-stokes equations, SIAM Journal on Scientific Computing 26 (2005) 1485-1503.

[22] H. Zheng, Y. Hou, F. Shi, L. Song, A finite element variational multiscale method for incompressible flows based on two local Gauss integrations, Journal of Computational Physics 228 (2009) 5961-5977.

[23] D. Sondak, J. N. Shadid, A. A. Oberai, R. P. Pawlowski, E. C. Cyr, T. M. Smith, A new class of finite element variational multiscale turbulence models for incompressible magnetohydrodynamics, Journal of Computational Physics 295 (2015) 596-616.

[24] V. John, S. Kaya, W. Layton, A two-level variational multiscale method for convectiondominated convection-diffusion equations, Computer Methods in Applied Mechanics and Engineering 195 (2006) 4594-4603.

[25] J. Wu, D. Gui, D. Liu, X. Feng, The characteristic variational multiscale method for time dependent conduction-convection problems, International Communications in Heat and Mass Transfer 68 (2015) 58-68.

[26] J. Wu, P. Huang, X. Feng, A new variational multiscale FEM for the steady-state natural convection problem with bubble stabilization, Numerical Heat Transfer, Part A: Applications 68 (2015) 777-796.

[27] E. Coenen, V. Kouznetsova, E. Bosco, M. Geers, A multi-scale approach to bridge microscale damage and macroscale failure: A nested computational homogenization-localization framework, International Journal of Fracture 178 (2012) 157-178.

[28] Y. Cong, S. Nezamabadi, H. Zahrouni, J. Yvonnet, Multiscale computational homogenization of heterogeneous shells at small strains with extensions to finite displacements and buckling, International Journal for Numerical Methods in Engineering 104 (2015) 235-259.

[29] V. D. Fachinotti, S. Toro, P. J. Sánchez, A. E. Huespe, Sensitivity of the thermomechanical response of elastic structures to microstructural changes, International Journal of Solids and Structures 69 (2015) 45-59. 
[30] F. Feyel, A multilevel finite element method $\left(\mathrm{FE}^{2}\right)$ to describe the response of highly nonlinear structures using generalized continua, Computer Methods in applied Mechanics and engineering 192 (2003) 3233-3244.

[31] F. Feyel, J.-L. Chaboche, $\mathrm{FE}^{2}$ multiscale approach for modelling the elastoviscoplastic behaviour of long fibre $\mathrm{SiC} / \mathrm{Ti}$ composite materials, Computer Methods in Applied Mechanics and Engineering 183 (2000) 309-330.

[32] V. Kouznetsova, M. G. Geers, W. M. Brekelmans, Multi-scale constitutive modelling of heterogeneous materials with a gradient-enhanced computational homogenization scheme, International Journal for Numerical Methods in Engineering 54 (2002) 1235-1260.

[33] T. Belytschko, S. Loehnert, J.-H. Song, Multiscale aggregating discontinuities: A method for circumventing loss of material stability, International Journal for Numerical Methods in Engineering 73 (2008) 869-894.

[34] I. Özdemir, W. Brekelmans, M. Geers, Computational homogenization for heat conduction in heterogeneous solids, International Journal for Numerical Methods in Engineering 73 (2008) $185-204$.

[35] E. Monteiro, J. Yvonnet, Q.-C. He, Computational homogenization for nonlinear conduction in heterogeneous materials using model reduction, Computational Materials Science 42 (2008) $704-712$.

[36] I. Özdemir, W. Brekelmans, M. G. Geers, FE² computational homogenization for the thermomechanical analysis of heterogeneous solids, Computer Methods in Applied Mechanics and Engineering 198 (2008) 602-613.

[37] A. Sridhar, V. G. Kouznetsova, M. G. Geers, Homogenization of locally resonant acoustic metamaterials towards an emergent enriched continuum, Computational Mechanics 57 (2016) 423-435.

[38] W. E, B. Engquist, Z. Huang, Heterogeneous multiscale method: a general methodology for multiscale modeling, Physical Review B 67 (2003) 092101.

[39] B. Engquist, Y.-H. Tsai, Heterogeneous multiscale methods for stiff ordinary differential equations, Mathematics of Computation 74 (2005) 1707-1742.

[40] M. Kamiński, Homogenization of transient heat transfer problems for some composite materials, International Journal of Engineering Science 41 (2003) 1-29.

[41] MATLAB R2016a, The MathWorks Inc., Natick, Massachusetts, United States, 2016.

[42] J. A. Dantzig, M. Rappaz, Solidification, EPFL press, 2009. 
[43] W. Sames, F. List, S. Pannala, R. Dehoff, S. Babu, The metallurgy and processing science of metal additive manufacturing, International Materials Reviews (2016) 1-46.

[44] N. Provatas, K. Elder, Phase-field methods in materials science and engineering, John Wiley \& Sons, 2011.

[45] H. Hashemi, C. Sliepcevich, A numerical method for solving two-dimensional problems of heat conduction with change of phase, in: Chem. Eng. Prog. Symp. Series, volume 63, pp. 34-41. 